\title{
IV. \\ Die münzmetrologischen Anhaltspunkte für die Erkenntnis der altfriesischen Ständeverfassung.
}

\author{
Von \\ Herrn Hugo Jaekel \\ in Breslau.*)
}

In meinen Aufsätzen „Êtheling, Frîmon, Frîling und Szêremon" und "Weregildus und simpla compositio" habe ich mich bemüht, aus authentischen Quellenzeugnissen und sicheren Ergebnissen der Münzmetrologie unverrückbare Anhaltspunkte für die Beurteilung der friesischen Standesverhältnisse zu gewinnen. ${ }^{1}$ ) Dieser Versuch, die Ständekontroverse wenigstens von einem Teile der Hypothesengespinste zu säubern, welche sich neuerdings an sie angesetzt haben, hat auf seiten Philipp Hecks einen Sturm der Entrüstung gegen die "Exaktheitsfanatiker" entfesselt. Die Entrüstung ist begreiflich. Denn wenn es gar dahin kommen sollte, daß der willkürlichen Hypothese eine geringere Bedeutung für die Entscheidung wissenschaftlicher Streitfragen beigemessen würde als dem exakten Beweise, wo bliebe dann Hecks Ständetheorie?

Um auch meine Auffassung der friesischen Standesverhältnisse als eine Verirrung zu erweisen, hat Heck nicht nur alle Register seiner Dialektik gezogen und seine sämtlichen Interpretationskünste spielen lassen, sondern auch die

*) Nachstehende Untersuchung ist der Redaktion bereits Ende Februar 1908 zugegangen, konnte aber wegen Raummangels im letzten Hefte, das als Jubiläumsband zur hundertjährigen Jubelfeier der Eichhornschen Rechtsgeschichte gedacht war, keine Aufnahme mehr finden und wurde deshalb im Einverständnis mit dem Verfasser für den vorliegenden Band zurückgelegt.

1) Vgl. in dieser Zeitschrift XXVII S. $275 \mathrm{ff}$, und XXVIII S. $102 \mathrm{ff}$. 
bisher von ihm aufgestellten numismatischen und metrologischen Einzelhypothesen zu einem kunstvollen Ganzen versponnen und schließlich seine rechtsgeschichtlichen Beweisgründe durch eine Verbindung mit rein philologischen unwiderstehlich gemacht. Als Frucht dieser Verbindung ist eine sehr umfangreiche Abhandlung erschienen: „Die friesischen Standesverhältnisse in nachfränkischer Zeit von Philipp Heck mit sprachwissenschaftlichen Beiträgen von Theodor Siebs" ${ }^{\text {". }}{ }^{1}$ )

Wenn ich trotz der peinlichen Entdeckungen, welche ich bei der Nachprüfung der Heckschen Beweisführung wieder machen mußte, den Handschuh aufhebe, so geschieht dies nur, weil auch von anderer Seite gegen meine Ergebnisse Einwendungen erhoben worden sind ${ }^{2}$ ) und deswegen eine noch festere Fundamentierung meiner Beweise und eine Betrachtung des "Sieges", den Heck davongetragen zu haben behauptet, angebracht sein dürften.

Den größten Teil der Heckschen Abhandlung nimmt der Nachweis ein, daß nicht die Compositio des homo liber, sondern die des homo nobilis der Lex Frisionum in der Normalkomposition der späteren friesischen Rechtsquellen fortlebe. Es wird nur weniger Sätze bedürfen, um den riesigen Hypothesenbau, durch welchen dies bewiesen werden soll, zum Einsturze zu bringen. Eingehender muß dagegen Hecks Widerlegung meiner Beweise für die Fortdauer der ständischen Tripartitio während des Mittelalters gewürdigt werden, weil sie sich nicht auf bloße Hypothesen stützt. Heck leitet diese Widerlegung mit weit ausgesponnenen allgemeinen Betrachtungen ein, die für den Gegenstand des Streites ohne entscheidende Bedeutung sind und hier übergangen werden können. Erst in $\$ 3$ geht er auf das eigentliche Thema über. Er behandelt hier den Szêremon, um dann in $\$ \S 4-6$ meine Beweise für den Wergeldvorzug des Edelings abzutun. Um uns so schnell als möglich eine Vorstellung von den Mitteln zu bilden, durch welche er meine auf bestimmte Quellenzeugnisse und unumstößliche metrologische Tatsachen gestützten Beweise niederzukämpfen vermocht hat, prüfen wir zuerst seine Besprechung der

1) Tübingen 1907 (Festgabe für Thudichum S. 47-230). - ${ }^{2}$ ) Von Rudolf His in dieser Zeitschrift XXVIII S. $439 \mathrm{f}$. und $442 \mathrm{f}$. 
Rüstringer Bußtaxen ( $\$$ 5, S. 91-97), weil er gerade durch diese Besprechung die "Unglaublichkeit" meines Verfahrens schlagend nachgewiesen zu haben meint.

\section{§ 1. Das jechtige Geld der Rüstringer BuBtaxen.}

Die in zwei friesischen und mehreren niederdeutschen Texten erhaltenen Rüstringer Bußtaxen ${ }^{1}$ ) bilden ein kleines Register, das, wie ich gezeigt habe ${ }^{2}$ ) und bereits His erkannt hatte ${ }^{3}$ ), seinen Bußberechnungen ein jechtiges Geld (Vollgeld) von 60 Schillinggewichten Gold zugrunde legt. Dieser Betrag war, solange Gold zu Silber wie 12:1 stand, mit 60 Geldmark gleichwertig. Die Geldmark (= 144 Rednath- oder Kawingpfennigen) stellte die Summe von 48 altfriesischen Silberdenaren dar. $\left.{ }^{4}\right)$ Somit betrug das jechtige Geld der Rüstringer Bußtaxen $60 \times 48$ oder $12 \times 240$ altfriesische Silberpfennige oder, da das altfriesische Denargewicht zum älteren karolingischen (Pippinischen) wie 11:12 stand ${ }^{5}$, $11 \times 240$ ältere karolingische Pfennige. Es deckte sich also mit dem Betrage, auf welchen in Lex Fris. XV, das Edelingsgeld angegeben wird: Compositio hominis nobilis librae XI per veteres denarios. Folgerichtig erklärte ich die Rüstringer Bußtaxen für Edelingstaxen.

Um gegen meine Beweisführung ankämpfen zu können, sucht Heck zunächst seinen Lesern eine Ungeheuerlichkeit einzureden. Bekanntlich sind in den beiden Rüstringer Rechtshandschriften ganz so wie in anderen friesischen Rechtshandschriften undatierte Stücke verschiedenen Inhalts und verschiedener Entstehungszeit vereinigt. Die ältesten rühren, abgesehen von den Zehn Geboten, nachweisbar aus dem 11., die jüngsten aus dem 13. Jahrhundert her. Unter diesen ganz heterogenen Stücken finden sich auch unsere

1) Nicht zu verwechseln mit dem Rüstringer Texte der Allgemeinen BuBtaxen! - ${ }^{2}$ ) Zeitschr. XXVII S. 303. - ${ }^{3}$ ) Strafrecht der Friesen im Mittelalter (1901) S. 228 und 292. Was die Berechnung des jechtigen Geldes der Rüstringer Bußtaxen aus den Lähmungsbußen dieses Registers angeht, so stellt mich Heck (S. 94) als den Verführten, His als Verführer hin. Ich brauche wohl kaum zu versichern, daß His an meinen Berechnungen der friesischen Wergelder völlig unschuldig ist. - $\left.{ }^{4}\right) \mathrm{Vgl}$ die Bestimmung des Silberwertes der Geldmark unten in §5. - s) Vgl. den Nachweis unten S. $60 \mathrm{f}$. 
Bußtaxen. Es finden sich darin ferner, aber getrennt von den Rüstringer Bußtaxen und auch nur in der einen Handschrift, zwei Rüstringer Volkssatzungen, nämlich die älteren und die neueren Rüstringer Küren. Es findet sich sodann in beiden Handschriften, aber getrennt von den Rüstringer Bußtaxen, eine aus Rüstringen stammende private Zusammenstellung über das "friesische" Recht. Die beiden Satzungen und die Privataufzeichnung, welche nach einem jechtigen Gelde von 40 Geldmark rechnen, also nur für den Stand der Gemeinfreien bestimmt gewesen sein können, verwenden als Hauptgeldwerte zwei Silber-Rechnungsmünzen, nämlich die Geldmark und die Reilmark. Dagegen rechnen die von diesen drei Stücken völlig getrennten Rüstringer Bußtaxen hauptsächlich nach Schillinggewichten Gold und Pfenniggewichten Gold. Heck will uns nun einreden, daß sich jene drei, nach Silbergeld rechnenden Stücke zu den von ihnen getrennten und noch nach Goldgeld rechnenden Bußtaxen etwa so verhalten hätten wie in einem modernen Gerichtskostengesetze die Gebührenordnung zu einer beigefügten Kostentabelle, daß also die vier Stücke als eine große Bufvorschrift anzusehen seien, die sich in Beschlüsse und Taxen gegliedert habe. Meine Ansicht, daß die Rüstringer Bußtaxen Edelingstaxen seien, die drei anderen Stücke dagegen für den Stand der Gemeinfreien berechnet wären, würde also nach seiner Meinung "inhaltlich der Annahme entsprechen, daß in 2 (wahrscheinlich amtlich herausgegebenen und benutzten) Exemplaren des Kostengesetzes die Gebührenordnung sich nur auf den Zivilprozeß bezieht und die Tabelle nur auf den Strafprozeß, ohne daß dies äußerlich erkennbar gemacht wäre" (S. 92). Wie niedrig muß Heck die Urteilskraft seiner Leser einschätzen, um ihnen mit einem solchen Vergleiche zu kommen ${ }^{1}$ ), den er hoffentlich selbst für völlig unzutreffend hält!

1) Auf S. 94 spinnt Heck den sonderbaren Vergleich noch weiter aus. In den Gött.Gel. Anz. 1902 S. 861 verlegte er die Rüstringer Küren und die jüngeren Bestandteile der Handschrift von 1327 in das 13., die Bußtaxen dagegen und die Privataufzeichnung über das „friesische" Recht in das 12. Jahrhundert! Im Jahre 1902 würde er also nicht umbin gekonnt haben, einen Vergleich, wie er ihn jetzt in seiner Ratlosigkeit zieht, als puren Unsinn zu bezeichnen. 
Die Höhe des jechtigen Geldes, nach welchem die Rüstringer Bußtaxen rechnen, bestimmte ich ${ }^{1}$ nach der Buße, welche sie auf die völlige Lähmung des Armes setzen. Ich sagte wörtlich: „Diese Bußtaxen lassen ganz so wie die Lex Frisionum das völlig gelähmte Glied halb so hoch büßen wie das völlig abgeschlagene", und ich begründete diesen Satz mit dem Hinweis auf die Fingerbußen in Fries. Rq. 120 und $537 \$ 9$. Heck aber schweigt sich über diesen Hinweis aus, um (S. 93) seinen Lesern erzählen zu können: „His und Jaekel setzen voraus (!), daß in Rüstringen die höchste Verstümmelung (!) immer (!) nur mit einem Viertel des Wergeldes gebüßt wurde." 2) Erst auf der folgenden Seite, wo er sich gegen meine "Entdeckungen" ganz besonders erhitzt, kommt er auch auf die Lähmung der Finger zu sprechen. Die Rüstringer Bußtaxen, meint er hier, "enthalten verschiedene Lähmungsbußen. Für Arm und Bein sind drei Grade angegeben mit den Bußen 15, $7_{1 / 2}^{1 / 2}$ und $3^{3 / 4}$ sc. $\left(1: 1_{2}^{1 / 2}:{ }^{1 / 4}\right)$, bei den Fingern begegnen vier Grade, z. B. $71 / 2,3^{3 / 4}, 1^{7} \mathrm{z}$, 15/16." Man traut beim Lesen dieser. Worte kaum seinen Augen. In beiden Texten der Bußtaxen sind für Arm und Bein $\mathrm{zwei}$ Lähmungsbußen angegeben, nämlich 15 Schillinggewichte Gold für völlige, $7^{1 / 2}$ Schillinggewichte Gold für teilweise Lähmung. Außerdem wird auf die bloße Schädigung eine Buße von $3^{3 / 4}$ Schillingsgewichten Gold und auf das völlige Abschlagen die Hälfte eines jechtigen Geldes gesetzt. Was aber speziell die Fingerbußen angeht, so leißt es hinsichtlich des Daumens und des kleinen Fingers im Oldenburger Texte: Thes im Text von 1327: Thes halhalderes and thes slutteres eider deres and thes sluteres eider thes gersfal achtunda half gersfal achtumda half skilling skilling goldis; stîuande and goldes; stêvande and stâtfiuwor stâk fiuwer skillinga wicht skillinga goldes bûta thrium goldis; helpande and haldande panningon wicht goldes; hiltwira skillinga wicht goldis pande ac haldande twira skilôni ôther halua pannig wicht linga goldes bûta ôr halua goldis; send se wrak, andlofta panning wicht goldes, thet is mir her.

1) Zeitschr. XXVII S. 303. - 2) Die Ausrufungszeichen rühren vou 
half pannig wicht goldis (Fries. achtunda half lâd; send se Rq. 120). swart, andlofta half panning wicht goldes, thet is threttinda half lâd (Fries. Rq. $537 \S 9$ ). Von vier Lähmungsgraden sagt die Stelle nichts, sondern sie kennt nur zwei Lähmungsbußen und außerdem je eine Buße für das Abschlagen und für die Beschädigung; und zwar soll nach ihr das völlig abgeschlagene Glied genau doppelt so hoch gebüßt werden wie das völlig gelähmte!

Es besagt ferner hinsichtlich der drei Mittelfinger der Oldenburger Text: Tha der Text von 1327: Tha thrê thrê fingera midda there hond fingra midda there hond alreck allerek fif skillinga wicht ...thredda halfskilling goldes; goldis; send se stêuande and hilpande and haldande alrek stâk, allerek thredda half fîftine panninga wicht goldes, skilling wicht goldis; helpande thet send fîf lâd; send se swart, and haldande fiftine pannig andloftha half panning wicht wicht goldis; wrac allerek ên goldes, thet is thredde half lâd achtunda half pannig wicht (Fries. Rq. 537 \& 9). goldis (Fries. Rq. 120).

Auch hier steht von vier Lähmungsgraden nichts, sondern es werden zwei Lähmungsbußen und je eine Buße für das $\mathbf{A b -}$ schlagen und für die Beschädigung aufgeführt; und auch nach dieser Stelle soll das völlig abgeschlagene Glied genau doppelt so hoch gebüßt werden wie das völlig gelähmte!1)

Heck hat also zuerst verschwiegen, daß ich mich für meine Aulfassung auf die Fingerbußen der Rüstringer Bußtaxen berufe, sodann meine Behauptung verdreht und schließlich den handschriftlichen Bericht über jene Fingerbußen so weit vergewaltigt, daß er ihn gegen meine Auffassung ins Feld führen konnte!

"Keine der beiden Rüstringer Bußtaxen", so erklärt Heck mit Nachdruck, "sagt etwas über die Relation der Lähmungsbußen zum Wergelde."

1) Wenn die drei Mittelfinger mit je 5, Daumen und kleiner Finger mit je $7 \frac{1}{2}$ Schillinggew. Gold zu bezahlen waren, so hatte man das Abschlagen aller fünf Finger mit 30 Schillinggew. Gold zu büßen. Bei den Ostfriesen wurde im Mittelalter, wie auch Heck weiß, die Gesamtheit der fünf Finger genau so hoch wie die Hand und diese mit einem 
Wer würde sich wohl beim Lesen dieser bestimmten Erklärung träumen lassen, daß sich die Sache genau umgekehrt verhält, daß die Relation der Lähmungsbußen zum Wergelde in beiden Texten der Bußtaxen ausdrücklich und auf das allerbestimmteste angegeben ist! Man liest wörtlich im Oldenburger Texte: $S a$ im Texte von 1327: $S a$ hwa hwa sa enne mon tô dêta sa enne mon thô dâda sleith, sleith, sa skillere sex hâud- sa skilre sex hâuedlessna thô lesna sella to fretha. Wndath fretha sella. Wndath hine alsa hine alsa fir, thet hine lemith, fì, thet hine lemi, fintma him fintma him ên fiardandêl ieldis $\hat{e} n$ fiardendèl $\hat{e}$ es ieldes thô tôbôte, sa skillere ên fiardandêl bôte, sa skil hi ên fiardandêl ênis dâddolgis tô fretha sella, ênes $\left.{ }^{1}\right)$ dâdolges fretho sella, thruch thet, thetter alilinath thruch thet theter aliknath lith lith with lif; fintma him ên with lif; fintma him enne achtunda dêl êris ieldis, sa is achtunda dêl ênes dâdolges, thi achtunda dêl ênes dâddolges ... alle dêde the ma dêth thi fretho. Alle tha deda, ther binetha lemithon usw. (Fries. edên send binetha lemithe usw. Rq. $538 \S 12$ ).

(Fries. Rq. 121).

Deutlicher als hier kann nicht gesagt werden, daß die Rüstringer Bußtaxen nur zwei Lähmungsgrade kannten und daß bei der völligen Lähmung eines Hauptgliedes $1 / 4$ Geld als Buße und als Friedensgeld ${ }^{1 / 4}$ vom Totschlagsfredus, bei der teilweisen Lähmung 1/8 Geld als Buße und als Friedensgeld $1 / 8$ vom Totschlagsfredus zu zahlen war.

Hat Heck diese Angabe nicht gekannt, d. h. hat er über die Rüstringer Bußtaxen geschrieben, ohne sich diese im Richthofenschen Abdruck wenig mehr als zwei Seiten einnehmende Aufzeichnung anzusehen? Oder liegt eine bewußte Entstellung der Wahrheit vor? Die Antwort kann nicht zweifelhaft sein. Heck hat die Stelle gekannt. In der Anmerkung $3 \mathrm{zu}$ S. 95 sagt er in Beziehung auf den Text von 1327: "Am Schlusse stehen noch die alten Lähmungsbußen." Ferner sagt er S. 223 wörtlich: „Die Rüstringer Bußtaxen bestimmen: 'Wer einen Mann zu Tode schlägt, der

halben 'Gelde' gebüßt. Das 'Geld'. nach welchen die Rüstringer Bußtaxen rechnen, betrug demnach 60 Schillinggew. Gold!

1) So ist statt fiardandeles zu lesen. 
soll 6 Hauptlösungen als Friedensgeld zahlen.' Bei den Lähmungen soll das Friedensgeld der Abstufung der Privatbußen entsprechen. Bei allen Taten, die geringer sind als Lähmungen, sind 10 Reilmark als Friedensgeld zu zahlen." In der dazu gehörigen Anmerkung gibt er sogar den friesischen Wortlaut der Stelle, aber mit Weglassung der beiden Sätze über die Lähmungsbuße! Ein Übersehen der Stelle liegt also nicht vor. ${ }^{1}$ ) Heck hat gewußt, daß in beiden Texten der Rüstringer Bußtaxen die Relation der Lähmungsbußen zum Wergelde ausdrücklich und auf das bestimmteste (1/4 Geld und 1/8 Geld) angegeben ist, und gleichwohl behauptet: „Keine der beiden Rüstringer Bußtaxen sagt etwas über die Relation der Lähmungsbußen zum Wergelde."

Heck schließt sein gewissenhaftes Referat über die Lähmungsbußen der Rüstringer Bußtaxen: „Unter diesen Umständen ist es eine reine Willkür, anzunehmen, daß die oberste Lähmungsbuße gerade $1 / \downarrow$ Wergeld sein müsse. Die Lähmungsbußen sind schon differenziert, die alte Einheitsbuße ist verschwunden. Den vollen Beweis dieser Wilkür erbringt nun eine Satzung, welche die Lähmungsbußen ändert und nur in der Rechtshandschrift von 1327 enthalten ist." Heck will also aus einem nicht zu den Bußtaxen gehörenden Satze, der sich nur in der jüngeren Handsehrift findet, mithin jungen Datums ist, und der, wie auch Heck erkannt hat, die älteren Lähmungsbußen ändert, nachweisen, daß meine Beurteilung der älteren Lähmungsbußen auf reine Willkür hinausläuft. Einen Nachweis dieser Art kann man natürlich dann führen, wenn die in Frage kommende Stelle, wie es hier tatsächlich der Fall ist, noch einen Schluß auf die ältere Ordnung der Lähmungsbußen erlaubt.

1) Daß Heck die Stelle schon seit langem uud genau kennt, ersieht man auch aus den Gött. Gel. Anz. 1902 S. 867, wo er sie wörtlich wie folgt überträgt: „Wer einen Mann zu 'Tode schlägt, soll 6 Hauptlösungen als Fredus zahlen. Verwundet er ibn so weit, daß eine Lähmung vorliegt, und findet man ihm ein Viertel des Wergelds zur Buße, so soll er $1 / 4$ des Totschlagsfredus als Fredus zahlen, deshalb weil Glied und Leben in Beziehung stehen. Findet man den achten Teil eines Wergelds, so beträgt der Fredus $1 / 8$ des T'otschlagsfredus. Bei allen Taten, die unterhalb der Lähmung stehen, ist der Fredus 10 Reilmark." 
Während das ältere Rüstringer Recht, wie wir sahen, nur zwei Lähmungsgrade kannte, werden in jener jüngeren Stelle (Fries. Rq. $542 \S 48$ ) deren drei unterschieden. Sie setzt auf das Abschlagen eines der sechs Glieder 20 Mark Buße und 60 Mark Friedensgeld, auf die höchste Lähmung 15 Mark Buße und 30 Mark Friedensgeld, auf die mittlere 10 Mark Buße und 2 Mark Friedensgeld, auf die geringste 5 Mark Buße und 1 Mark Friedensgeld. Da die Stelle das Abschlagen eines Hauptgliedes mit 20 Mark büßen läßt, muß sie mit einem "Gelde" von 40 Mark, womit selbstverständlich Geldmark gemeint sind, gerechnet haben. Heck meint nun, daß die Stelle beweise, daß eine höchste Lähmungsbuße zum Betrage von 15 Mark mit einem Gelde von 40 Mark vereinbar sei, daß also, wenn diese höchste Lähmungsbuße von 15 Mark auch in den Rüstringer Bußtaxen begegne, "daraus nicht gefolgert werden könne; daß die Bußtaxen eine andere Wergeldzahl voraussetzen als die Satzungen". Hier liegt eine handgreifliche Verdrehung der Streitfrage vor. Es hat ja niemand bestritten, daß nach der jüngeren Rüstringer Ordnung der Lähmungsbußen die auf 15 Mark festgesetzte höchste Lähmungsbuße neben einem "Gelde" von 40 Mark stand. Wohl aber wird von mir bestritten, daß dies mit der älteren Rüstringer Ordnung der Lähmungsbußen vereinbar war, weil nach dieser die höchste Lähmungsbuße 1/+ Geld betrug. Heck hätte also, um mich zu widerlegen, prüfen sollen, ob jene Stelle nicht einen Schluß auf die ältere Bußordnung gestattet, die durch sie abgeändert wurde. Dies ist in der Tat der Fall. Sie stellt nämlich dem halben "Gelde" (20 Mark) ein Friedensgeld von 60 Mark, der höchsten Lähmungsbuße (15 Mark) dagegen ein solches von 30 Mark zur Seite, d. h. einen Friedensgeldbetrag, der einem Viertelgelde entsprach. Erklärlich ist dies nur, wenn nach der älteren Ordnung die höchste Lähmungsbuße 1/t Geld betrug! Der Sachverhalt ist so einfach und klar, daß man sich unwillkürlich fragt, ob ihn Heck wirklich nicht durchschaut oder ob er es nur für nicht opportun gehalten hat, auf ihn hinzuweisen. Um die richtige Antwort zu finden, lesen wir, was Heck am Schlusse seiner Abhandlung (S. 227 Anm. 1) über die jüngere Rüstringer 
Ordnung der Lähmungsbußen anmerkt: „Die Ordnung ist nur insofern gestört, als bei den beiden obersten Gliederbußen die Privatbußen 20 und 15 sich anders verhalten als die Friedensgelder 60 und 30. Diese Differenz ist unmittelbar bezeugt und erklärt sich dadurch, daß die Abstufung der Privatbußen geändert wurde. Die Abstufung der Friedensgelder entspricht dem alten Verhältnisse der Privatbußen." Heck ist also ganz so wie ich davon überzeugt, daß nach der älteren Rüstringer Bußordnung die höchste Lähmungsbuße ${ }^{1 / 4}$ Geld betrug und daß diese Buße erst im 13. Jahrhundert auf $3 / 8$ Geld erhöht wurde!

An den sonstigen von Heck gegen meine Auffassung der Rüstringer Bußtaxen ins Feld geführten Beweisgründen vermißt man jede Spur von überzeugender Kraft.

Er bezeichnet den interpolierten jüngeren Text der Rüstringer Bußtaxen als ,eine Umarbeitung der älteren Bußtaxen" und konstruiert sich so "ältere" und "neuere" Rüstringer Bußtaxen. Die älteren hätten „für geringe Lähmungen die Buße von $71 / 2$ Schilling Goldgewicht gegeben, die in dem angeführten $\S 48$ nicht mehr vorkomme". In dem ersten Teile der neueren Bußtaxen sei nun am Anfange der Aufzeichnung zu diesen $7 \frac{1}{2}$ Schillingen neu hinzugefügt worden: „Das sind 5 Mark." Man habe also die Tabelle nach der neuen Ordnung zu korrigieren gesucht; dies wäre aber ganz unmöglich gewesen, wenn man die Taxen nicht auf dieselben Personen und dieselbe Zeit bezogen hätte wie die Satzungen. Heck scheint also noch nicht erkannt zu haben, daf die an 17 Stellen des jüngeren Bußtaxentextes mit thet send oder mit thet is an die alten Goldgeldbeträge angeschlossenen Lot- und Mark-Beträge nichts anderes als Reduktionen sind, daf lediglich eine Umrechnung von Goldgeld in Silbergeld vorliegt. Meint er wirklich, daß durch die Formeln thet send und thet is etwas anderes als die Gleichwertigkeit bezeichnet werden sollte? Dann ist es freilich um seine Kenntnis des altfriesischen Geldwesens noch trauriger bestellt, als ich dachte.

Nach Heck sollen die Allgemeinen Bußtaxen nach einer älteren Münze rechnen als die Rüstringer Bußtaxen. Gerade das Umgekehrte ist der Fall. Die Goldgeldrechnung der 
Rüstringer Bußtaxen ist älter als die Silbergeldrechnung der Allgemeinen Bußtaxen, denn auch in Friesland ist man im Mittelalter nicht vom Silber- zum Goldgelde, sondern vom Gold- zum Silbergelde übergegangen. Wahrscheinlich wollte Heck diesen klaren Sachverhalt dadurch verschleiern, daß er skilling wicht goldis und panning wicht goldis nicht durch "Schillinggewicht Gold“ und „Pfenniggewicht Gold", sondern fälschlich mit "Schilling Goldgewicht" und "Pfennig Goldgewicht" wiedergibt.

Daß die Allgemeinen Bußtaxen bei der Redaktion der Rüstringer Bußtaxen benutzt worden seien, ist eine Behauptung ins blaue hinein. ${ }^{1}$ ) Die beiden Register stehen, wie die Anordnung des Stoffes und die Bußenkasuistik auf den ersten Blick zeigen, einander völlig unabhängig gegenüber.

Meine Behauptung, daß die 60 Geldmark, welche das jechtige Geld der Rüstringer Bußtaxen ausmachte, mit den librae XI per veteres denarios, auf welche Lex Fris. XV die Compositio hominis nobilis festsetzt, gleichwertig seien, ist von dem Numismatiker Heck (S. 93 Anm. 3) mit apodiktischer Gewißheit verworfen worden:

„Die einzelnen Brücken dieser Schlußfolgerung sind 1. die Geldmark zählt 12 Schilling Redn. Münze. 2. Der Rednathpfennig ist $1 / 3$ des altfriesischen Pfennigs. 3. Die Pfunde in Tit. 15 sind Römerpfunde zu 240 Pippinischen Pfennigen. 4. Die Pippinischen Pfennige haben sich zu den altfriesischen verhalten wie $11: 12$. Alle 4 Annahmen sind unrichtig."

Ein hartes Urteil! Leider fehlt die Urteilsbegründung! Zur Entschuldigung meiner "Annahmen" kann ich nur meinen unheilbaren "Exaktheitsfanatismus" anführen.

1) Ebenso grundlos ist es, wenn Heck (Gött. Gel. Anz. 1902 S. 854) die Hunsegauer Bußtaxen neine Umarbeitung der Allgemeinen Bußtaxen" nennt und von einem Zusammenhange der Hunsegauer und der Allgemeinen Bußtaxen spricht. Er verrät nur seine völlige Unbekanntschaft mit der altfriesischen Geldrechnung, wenn er (Mitteilungen des Instituts für österreichische Geschichtsforschung VII S. 769) erklärt: „Die Hunsingoer Bußtaxen gehören in der Masse ihrer Bußzahlen frühestens in das 12. Jahrhundert und zwar eher in die zweite Hälfte als in die erste, möglicherweise aber auch in die erste Hälfte des 13." In der dazu gehörenden Anm. 4, die Hecks völlige Hilflosigkeit auf münzmetrologischem Gebiete offenbart, erlaubt er sich eine krasse Quellenvergewaltigung ( $v$ gl. unten S. 70). 
König Pippin hatte i. J. 754/755 verordnet, daß fortan aus einem römischen Pfunde Silber $(22 \times 12 \Rightarrow) 264$ Pfennige ausgebracht werden sollten. Andrerseits betrug Karls d. Gr. Münzpfund, aus dem 240 Pfennige geprägt wurden, genau $5 / 4$ röm. Pfund. ${ }^{1}$ ) Demnach standen der ältere und der jüngere karolingische Denar zueinander wie $1 / 264: \frac{1 \times 5}{240 \times 4}=$ $8: 11$. Nun verhielt sich nach Add. leg. Fris. III 73 der Denar der Ostfriesen, d. h. der altfriesische Denar, zum jüngeren karolingischen wie $2: 3$ oder wie $8: 12$. So ergab sich mir, daß der altfriesische Denar zum Pippinischen wie 11:12 gestanden hat.

$\mathrm{DaB}$ ich irgendwo die nichts weniger als exakte Behauptung aufgestellt haben sollte, daß die Pfunde des Tit. XV der Lex Frisionum „Römerpfunde zu 240 Pippinischen Pfennigen" seien, ist ausgeschlossen. Aus dem röm. Pfunde feinen Silbers sollten ja nach Pippins Verordnung 264 Pfennige ausgebracht werden. Der Pippinische Pfennig hätte also nur dann $1 / 240$ röm. Pfund gewogen, wenn er einen Zusatz von $1 / 11$ gehabt hätte, also zu $10 / 11$ fein gewesen wäre. Davon wissen aber die Quellen nichts. Ich habe etwas ganz anderes gesagt, nämlich daß mit der libra per veteres denarios des Tit. XV ein fränkisches Zählpfund zu 240 Pippinischen Denaren gemeint sei, weil die Lex Frisionum unter libra das fränkische Zählpfund von 20 Solidi zu je 12 Denaren versteht!

Daß die Geldmark 12 Schillinge zu je 12 Rednathpfennigen umfaßte und der Rednathpfennig $1 / 3$ vom altfriesischen Pfennige ausmachte, sind keine "Annahmen“, sondern, wie unten gezeigt werden soll ${ }^{2}$ ), sichere Ergebnisse der friesischen Münzmetrologie. Übrigens ist die Einteilung der Geldmark für die Frage nach dem Silberwert dieser Mark ganz gleichgültig, denn dieser Silberwert ist dadurch genau bestimmt, da $\$$ die friesischen Rechtsquellen 60 Geldmark mit 16 Mark Englisch, also mit $16 \times 144$ Sterlingen gleichsetzen. ${ }^{3}$ ) Es wog aber der Sterling im 13. Jahrhundert, dem diese Rechtsquellen angehören, 1/256 engl. Troypfund, und sein gesetzlicher Feingehalt betrug ${ }^{35} / 36$. Folglich enthielt damals die Mark Englisch $\left({ }^{144 / 256} \times{ }^{35 / 36}=\right)^{35 / 64}$ Troypfund reinen

1) Dies hat auch Heck erkannt; vgl. z. B. Gemeinfreie S. 148. 2) Vgl. unten S. $82 \mathrm{f}$. und $98 \mathrm{f}$. - $^{\text {s) }}$ Vgl. unten S..98f. 
Silbers. 16 Mark Englisch stellten also ${ }^{35 / 4}$ Troypfund oder, da 1 Troypfund $=8 / 7$ röm. Pfund, 10 röm. Pfund reinen Silbers dar. Sie waren demnach mit $10 \times 264$ oder $11 \times 240$ Pippinischen Denaren gleichwertig. Nach allem ist die Gleichsetzung der librae XI per veteres denarios des Tit. XV der Lex Frisionum mit 60 Geldmark keine willkürliche Hypothese, sondern das unanfechtbare Ergebnis einer richtig gestellten und exakt gelösten Rechenaufgabe.

Unsere vorstehende Erörterung dürfte über zweierlei volle Klarheit verbreitet haben, nämlich

1. über die Beschaffenheit der Waffen, mit welchen Philipp Heck für den Sieg der Wahrheit streitet, und

2. darüber, daß die Rüstringer Bußtaxen nach einem jechtigen Gelde von 60 Geldmark rechnen und daß dieser Betrag mit den librae XI per veteres denarios gleichwertig ist, auf die sich nach Lex Fris. XV die compositio hominis nobilis belief.

His sieht in den 60 Geldmark der Rüstringer Bußtaxen das "Geld" des Gemeinfreien. Er muß daher annehmen, daß das Geld der Freien später auf 40 Geldmark herabgesetzt worden ist. Allein es steht quellenmäßig fest, daß das ostfriesische Normalgeld seit der fränkischen Zeit wohl Erhöhungen, niemals aber eine Herabsetzung erfahren hat. Wenn es sich also im 12. und 13. Jahrhundert auf 40 Geldmark belief, so kann es vor dieser Zeit nicht 60 Geldmark betragen haben. Das „Geld", nach welchem die Rüstringer Bußtaxen rechnen, kann demnach nicht das der Gemeinfreien, sondern nur das einer über diesen stehenden Bevölkerungsschicht gewesen sein. Hierzu stimmt nun, daß die 60 Geldmark mit den 11 Pfund gleichwertig waren, welche nach Lex Fris. XV die compositio hominis nobilis ausmachten. Die aus dem 11. Jahrhundert stammenden Rüstringer Bußtaxen müssen also für den Edeling berechnet gewesen sein.

\section{§ 2. Das Fivelgauer Edelingsgeld zur Zeit Emos von Wittewierum.}

In $\S 4$ (S. $87 \mathrm{ff}$.) kämpft Heck gegen das Ergebnis an, welches ich hinsichtlich der ostfriesischen Standesverhältnisse aus zwei gleichzeitigen Fivelgauer Quellen, dem „Primum 
plebiscitum in Fywelgonia" und der Chronik Emos von Wittewierum gewonnen habe. ${ }^{1}$ ) Das Plebiscitum gibt das normale Friedensgeld zu centum marcae (120 Mark) an. Daraus schlob ich auf eine Normalkomposition von quadringentae marcae $(4 \times 120$ Mark), weil nach altostfriesischem Rechte das normale Friedensgeld zum Normalgelde wie $1: 4$ stand. $^{2}$ ) Heck wendet ein, daß „die Relation zwischen Friedensgeld uud Wergeld in den jüngeren Quellen keineswegs so konstant sei, daß bei einer Quelle ungewissen Datums dieser Schluß möglich wäre". Er möchte also in dem Leser den Glauben erwecken, da $B$ man in dem Primum plebiscitum Fivelgumanum möglicherweise eine junge Quelle zu sehen habe. Aus diesem Wunsche erklärt es sich auch, daß er diese Satzung konsequent als "Plebiscitum Fivelgoniae" anführt, also das davorstehende "primum " stillschweigend unterdrückt. Das Datum dieser Satzung läßt sich natürlich nicht mehr bis auf Jahr und Tag berechnen, wohl aber kann man einen bestimmten Zeitraum umgrenzen, in welchen sie fallen muß. His meinte ${ }^{3}$ ), daß das Plebiscitum jünger sei als die Hunsegauer Küren von 1252, aber noch dem 13. Jahrhundert angehöre. Dem widersprechen die Münzverhältnisse. In den Fivelgauer und Hunsegauer Rechtsquellen, welche dem Ausgange des 13. Jahrhunderts angehören, begegnen uns die Mark weißen Silbers, die Mark Englisch, die Groninger Mark und die münstersche Mark. Auch die Hunsegauer Küren von 1252 verwenden regelmäßig die "merk hwîtes selueres " und die "merk Grênengslachta". Nur in Abschnitten, welche augenscheinlich aus älteren Satzungen übernommen sind, nämlich in $\S \S 5,11,12,15,22$ und 29 , begegnet noch die „merk" schlechthin, $d . h$. die einheimische Mark. ${ }^{4}$ ) Diesen Münzverhältnissen der Rechtsquellen entspricht es, daß von den Wittewierumer Chronisten der Continuator Menconis die einheimische Mark gar nicht mehr und Menko sie nur noch in den älteren Partieen seiner Chronik verwendet, während Emo regelmäßig nach ihr rechnet.5)

1) Zeitschrift XXVII S. 301 f. - $\left.{ }^{2}\right)$ Vgl. Zeitschrift XXVIII S. 138141 und unten S. $77 \mathrm{ff},-{ }^{3}$ ) Strafrecht der Friesen S. 7. - $\left.{ }^{4}\right)$ Vgl. die Hunsegauer Küren in Fries. Rq. $329 \mathrm{ff}$. - ${ }^{5}$ ) Der Continuator Menconis 
Das Plebiscitum muß also, da es ausschließlich nach der "Mark" schlechthin, d. h. nach der einheimischen Mark, rechnet, vor der Mitte des 13. Jahrhunderts abgefaßt sein. Andrerseits geht aus $\S \S 15-17$ dieser Satzung hervor, daB zur Zeit ihrer Abfassung bereits Klöster im Fivelgau vorhanden gewesen sein müssen. Dies weist auf die Zeit nach den Fivelgauer Klostergründungen des beginnenden 13. Jahrhunderts. Somit kann es keinem Zweifel unterliegen, daß das Primum plebiscitum Fivelgumanum in die erste Hälfte des 13. Jahrhunderts gehört ${ }^{1}$ ) und nicht als eine "jüngere", sondern als eine alte Rechtsquelle zu gelten hat. Soll also aus dem Friedensgelde dieser Quelle (centum marcae $=$ 120 Mark) das damalige Normalgeld erschlossen werden, so kann dies nur nach der altostfriesischen Relation geschehen. Nach dieser stand der Totschlagsfredus zum Normalgelde wie $1: 4$. Das Normalgeld der Fivelgauer muß also zu der Zeit, als jenes Plebiscitum entstand, quadringentae marcac (= 480 Mark) betragen haben.

Aus unsrer Datierung des Primum plebiscitum Fivelgumanum folgt aber auch, daß es in die Zeit fällt, in welcher der Fivelgauer Abt Emo von Wittewierum (1170-1237) seine Chronik schrieb. Er tat dies in den Jahren 1219-1237.²) Plebiszit und Chronik gehören derselben Periode und demselben Gaue an. Wenn also beide die Mark, nach welcher sie rechnen, als "marca" ohne jeden Zusatz bezeichnen, so müssen sie mit dieser marca ein und denselben Wert meinen. Heck wendet ein, es habe in Friesland sehr verschiedene

rechnet nur nach Sterlingen und nach den Pfennigen von Münster und von Deventer (MG. SS. XXIII 564, 565, 566). Bei Menko begegnet die "Mark" schlechthin nur noch zweimal (MG. SS. XXIII 534 und 545); sonst rechnet er nach der Münze von Groningen (S. 534), von Deventer (S. 534), von Münster (S. 541) und nach Sterlingen (S. 541, 554, 560). Emo erwähnt die Emdener (S. 517) und die Groninger Münze (S. 511, 512), rechnet aber regelmäßig nach der "Mark" schlechthin (S. 476, $507,508,509,512,516)$.

1) Eine noch genauere Datierung dieser wichtigen Rechtsquelle werde ich bei andrer Gelegenheit versucheu. $\left.-{ }^{2}\right) \mathrm{V}$ gl. Aem. W. Wybrands, De abdij Bloemhof te Wittewierum in de dertiende eeuw (1883) S. 185, wo überzeugend dargetan ist, daß Emo erst im Jahre 1219 mit der Abfassung seiner Chronik begonuen haben kann. 
Marksorten gegeben. Dies hat noch niemand bestritten. Hier handelt es sich aber nicht um Friesland, sondern um ein und denselben kleinen Bezirk, das Fivelingalond, und um ein und dieselbe Zeit! Wenn Heck es für schlechthin unzulässig erklärt, die Mark, nach welcher Emo rechnet, mit der Mark, welche in dem Plebiscitum genannt wird, zu identifizieren, so müssen wir es auf Grund unsrer Datierung der Satzung umgekehrt für schlechthin unzulässig erklären, hier von zwei verschiedenen Marksorten zu reden.

Die marca des Chronisten hatte genau denselben Wert wie die marca der Satzung. Die quadringentae marcae also, auf welche nach dem Berichte Emos vom Jahre 1234 die Fivelgauer bei ihrer Auflehnung gegen die kanonischen Satzungen die compositio sacerdotis occisi herabgesetzt wissen wollten, stellten das damalige Fivelgauer Normalgeld dar. Dieses Ergebnis ist jedem Zweifel entzogen.

Daß auch in Friesland dem Priester, vorausgesetzt daß er seinen durch Gesetz näher bestimmten Sonderfrieden (afrethe) nicht eingebüßt hatte, ein sehr hohes Wergeld zukam, ist bekannt. ${ }^{1}$ ) Fiel der âfrethe fort, so kamen dem Priester nur das Wergeld und die Bußen des Gemeinfreien zu. Im $\& 35$ der Hunsegauer Bußtaxen wird ausdrücklich bestimmt: Al thet ther tha prêstere dên is buta tha engleska wềle and bûta houe, al ênbête ti bêtane. ${ }^{2}$ Auch ein mit schweren Sünden befleckter Lebenswandel muß nach friesischer Anschauung dem Priester den âfrethe genommen haben. Wenn also die Fivelgauer in ihrer Empörung über den befleckten Lebenswandel ihrer Geistlichen den BeschluB

1) Über die Wandlungen des friesischen Priesterwergeldes weiß Heck ebensowenig Bescheid wie über die des friesischen Laienwergeldes. Er bemerkt, daß nach dem älteren Hunsegauer wie nach dem Fivelgauer Rechte der Priester die achtfache Buße des Laien hatte (S. 88). Aber dies war in dor ersten Hälfte des 13. Jahrhunderts längst nicht mehr der Fall. Wie eine bei Emo (MG. SS. XXIII 516) erhaltene Urkunde aus dem Jahre 1228 ausdrücklich angibt, betrug damals "secundum consuetudinem terrae Fivelgoniae" das Priestergeld "septingentae marcae Groniensis monetae ", also $7 \times 120$ Groninger Mark, d. h. das $10^{1 / 2}$ fache des damaligen Fivelgauer Normalgeldes oder das 14fache der Compositio, welche Lex Fris. XV dem homo liber gibt. 2) Fries. Rq. 385 . 
beschworen, compositionem sacertotis occisi non excedere cccc marcas, und dabei iustam quandam querelam sibi habere videbantur adversus saccrdotes fornicarios et tabernarios, so kann es sich bei den ccec marcae nur um das Wergeld des Gemeinfreien handeln. Damit erledigt sich Hecks Einwand (S. 88), daß Emo ein von seinem Standpunkte ${ }^{1}$ ) aus so horrendes Vorgehen schwerlich verdunkelt, sondern wahrscheinlich gesagt haben würde: „non excedere compositionem laici“. Ob Emo "compositionem laici" oder "ccec mar'cas" sagte, kam auf genau dasselbe hinaus.

Das Primum plebiscitum Fivelgumanum und die Chronik Emos ergeben also zur Evidenz, daß in der ersten Hälfte des 13. Jahrhunderts im Fivelgau der normale Totschlagsfredus 120 und das Normalgeld $4 \times 120$,Nark" betragen hat; und es liegt auf der Hand, dal diese Fivelgauer "Mark", da zu derselben Zeit in Rüstringen der normale Totschlagsfredus 120 Reilmark und das Normalgeld $4 \times 120$ Reilmark $(=40$ Geldmark) betrug, denselben Wert wie die Rüstringer Reilmark des 13. Jahrhunderts gehabt haben muß.

Die unumstößliche Tatsache, daß zu Emos Zeit quingentae marcae $(5 \times 120 \mathrm{Mark})$ an compositio vitae und redemptio capitis zu zahlen waren, wenn ein Gemeinfreier einen Standesgenossen erschlagen hatte, ist für die Beurteilung des Betrages maßgebend, welchen nach demselben Emo der Propst des Fivelgauer Nonnenklosters Schildwolde im Jahre 1224 als compositio vitae und renlemptio monasterii zu zahlen bereit war, als bei der Belagerung dieses Klosters auf seiten der Angreifer ein Edeling (nobilis) getötet worden war. Es heißt von dem Propste: „Prccepit eis (den Verwandten des Erschlagenen) offerri, ut dicitur, mille marcas pro compositione vitae ipsius ot in redemptionem monasterii ut parcerent." Daß das Friedensgeld, wie Heck (S. 90) bemerkt, nicht an die Verwandten gezahlt wurde, ist selbstverständlich. Jene "redemptio monasterii" ist, wie sich ebenfalls von selbst versteht, der Betrag, welcher der Sippe der toten Hand angeboten wurde, um ihr "Brand und Bruch" des Klosters

1) Es will mir übrigens scheinen, als ob sich Heck den Standpunkt, welchen Emo dem Treiben der Fivelgauer Weltgeistlichkeit gegenüber einnahm, nicht allzu klar gemacht habe. 
abzukaufen. Dieser Betrag war in den Ommelanden dem vierten Teile der Privatbuße gleich ${ }^{1}$ ), machte also genau ebensoviel wie das Friedensgeld aus. Die Compositio und die "Brandpfennige" ergaben daher zusammen genau dieselbe Summe wie die um das Friedensgeld vermehrte Compositio. Wenn also die den Magen des Erschlagenen als compositio vitae und als redemptio monasterii angebotene Gesamtsumme mille narcas, betrug, so muß auch die Summe, auf welche gegebenenfalls Compositio und Fredus zusammen festzusetzen waren, mille marcae betragen haben. Da nun dieser Betrag $(10 \times 120$ Mark) genau das Doppelte von der Summe $(5 \times 120$ Mark) ausmachte, welche für einen erschlagenen Gemeinfreien an Compositio und Fredus zu zahlen war, so kann nur geschlossen werden, daß im 13. Jahrhundert der Edeling (nobilis) im Fivelgau doppelt soviel galt als der Gemeinfreie.

Der Edeling, dessen Tod man sühnen wollte, war beim Angriff auf ein Kloster durch die Hand eines Verteidigers gefallen. Das Kloster war also nach dem Rechte des Landes nicht verpflichtet, Vollgeld und Brandpfennige zu zahlen. Wenn sich der Propst des Klosters gleichwohl dazu bereit erklärte, so konnten seine Gegner darin nur ein Zeichen schimpflicher Schwäche oder eines bösen Gewissens sehen. Kein Wunder daher, daß der mit ihm bitter verfeindete Emo von Wittewierum den Vorgang, von dem er durch Hörensagen wußte, in seiner Chronik vermerkte. Durch ein hinzugefügtes "ut dicitur" gibt er deutlich zu verstehen, daß ihm selbst eine so wenig ehrenvolle Nachgiebigkeit des Schildwolder Propstes fast unglaublich erscheinen wollte. Daß sich Emo durch den Zusatz "ut dicitur" gegen den Vorwurf der Lüge habe schützen wollen, wie Heck (S. 90) wähnt, ist ausgeschlossen. Er wollte lediglich sagen, daß er einen Vorgang, von welchem sich die Leute allgemein erzählten, berichte. Ob der Propst wirklich volle Sühne angeboten hat oder ob ihm die Leute dies nur nachgesagt haben, ist für die rechts-

1) Vgl. die älteren Langewolder Küren $\$ 2$ (Fries. Rq. 366) und die jüngeren Langewolder Küren $\$ 7(\mathrm{Rq} .370)$. An beiden Stellen werden die "Brandpfennige“ auf 30 Mark festgesetzt, während das Normalgeld nhundert pont" oder, was damals dort dasselbe bedeutete, "hundert mark “, also 120 Mark betragen sollte! Man vergleiche wegen der „Brandpfennige" auch §14 der älteren Fredewolder Küren (Fries. Rq. 378). 
geschichtliche Verwertung des Berichtes gleichgültig. Für diese kommt nur die durch Emos Erzählung verbürgte Tatsache in Betracht, daß im Jahre 1224 im Fivelgau ein Edeling, der die Tötung eines Standesgenossen sühnen wollte, an die Sippe des Erschlagenen an Komposition und Brandpfennigen $10 \times 120$ Reilmark zu zahlen hatte.

\section{§ 3. Der Edeling der Hunsegauer Küren von 1252.}

Wie Heck S. $97 \mathrm{ff}$. eingehend darlegt, ist es ihm nunmehr auch geglückt, den wahren Sinn der Hunsegauer Edelingsstelle zu finden. Er mag aufgeatmet haben, als er diese böse Stelle endlich als eine bloß "scheinbare crux" bezeichnen konnte (S. 74). Denn über keinen Satz der friesischen Rechtsquellen hat sich der Entdecker der Standeseinheit, die während des Mittelalters in Friesland geherrscht, mehr den Kopf zerbrochen als über den $\S 13$ der Hunsegauer Küren ron 1252, dessen Inhalt und Ausdrucksweise sich als so deutlich und klar erwiesen, daß Heck keinen Angriffspunkt für seine Interpretationskunst erspähen konnte.

Umbe alle dîddele and umbe alle tâchnenga twisk thene êtheleng and thene mon, alsa hit êr was ${ }^{1}$ ) („Hinsichtlich aller Totschlagstaten und hinsichtlich aller Zwistigkeiten zwischen dem Edeling und dem Mann so, wie es früher war" ${ }^{\text {(") }}$.

Daß man unter einem êtheleng einen Edeling zu verstehen hat und $\mathrm{da} \mathrm{B}$ mon in den friesischen Rechtsquellen des 13. Jahrhunderts die technische Bezeichnung des normalfreien Friesen ist, für den eben jene Rechtsquellen berechnet sind, läßt sich nicht weginterpretieren. Die Verwendung der allgemeinen Gattungsbegriffe "der Edeling " und "der Mann" beweist, daß die Stelle von der Gesamtheit der Edelinge und der Gesamtheit der Gemeinfreien etwas aussagen will. Die Gleichsetzung des êtheleng und des mon verbietet sich, weil über das Verhältnis twisk thene êtheleng and thene mon eine Bestimmung getroffen wird. In welcher Beziehung das Verhältnis zwischen dem Edeling und dem Gemeinfreien geregelt werden soll, ist durch die Worte umbe alle dâddele and umbe alle tâchnenga unzweideutig gesagt. Die Wendung endlich,

1) Fries. Rq. 329. 
durch welche die Bestimmung dieses Verhältnisses ausgesprochen wird, "so, wie es früher war", läßt an Deutlichkeit nichts zu wünschen übrig. Man sollte also meinen, daß kein Forscher anders schließen kann, als daß $\$ 13$ der Hunsegauer Küren das althergebrachte Rechtsverhältnis zwischen zwei ganzen Bevölkerungsschichten, von denen die eine durch die Gesamtheit der Edelinge, die andere durch die Gesamtheit der Gemeinfreien gebildet wurde, also das Rechtsverhältnis zweier Stände aufrechthalten wollte, d.h. daß im 13. Jahrhundert im Hunsegau die Edelinge einen Stand oberhalb der Gemeinfreien bildeten. Heck aber ist jetzt dahinter gekommen, daß aus der Stelle etwas ganz anderes herauszulesen sei. Seiner Interpretation kann wenigstens das Prädikat "originell" nicht versagt werden.

Zunächst eskamotiert Heck aus der Stelle die beiden Gattungsbegriffe, indem er den friesischen Text in die Anmerkung verweist und seiner Erörterung eine Übersetzung zugrunde legt, in welcher die Worte twisk thene êtheleng and thene mon durch "zwischen den Edelingen und den Leuten" wiedergegeben sind. Sodann soll sich der \& 13 nach ihm nicht wie die anderen Paragraphen der Küren auf Dinge und Verhältnisse, welche eintreten werden, sondern auf Vergangenes beziehen. Es liege eine Übergangsvorschrift vor, welche besage: „Alle jene Totschlagstaten und alle jene Ereignisse, welche zwischen den Edelingen und den Leuten vorgekommen sind, sollen nach dem bisherigen Rechte beurteilt werden." Die Stelle beziehe sich auf die Zusammenstöße, welche die Fivelgauer und Hunsegauer in den Jahren 1250 und 1252 mit Groningen hatten, und es seien "unter den Edelingen die Ritter von Groningen und Pedese (also keine Friesen!) zu verstehen". Darüber, daß die friesische Bezeichnung des Ritters nicht êtheling, sondern ridder ist, setzt er sich leicht hinweg. Das Wort „edel" sei zu jener Zeit, wie die Chronik von Wittewierum zu ergeben scheine, im täglichen Sprachgebrauch schon mit Beziehung und Beschränkung auf sozial höherstehende Elemente gebraucht worden. $\mathrm{Daß}$ es sich in jenem $\S 13$ nicht um den ,täglichen Sprachgebrauch", sondern nur um die Sprache des Gesetzes handeln kann, wird von Heck nicht in Erwägung gezogen. 
„Die Auffassung der Edelinge als einer außerhalb der Landesgemeinde stehenden Personengruppe wird", meint Heck, "dadurch unterstützt, daß die Gegenseite einfach als die "mon" bezeichnet ist." Mon heiße einfach Mann. Mensch. Der Plural sei mit "the liude" (sic!) ganz gleichbedeutend. ${ }^{1}$ ) Es bezeichne also ,mon " in den fraglichen Küren jeden Landgenossen ohne ständischen Nebensinn, ohne Abschluß nach oben. Hätte sich die Stelle ex professo mit dem Unterschiede der Stände innerhalb der Landgenossen beschäftigt, so hätten für beide Teile spezielle Ausdrücke, also etheling und friling, gesetzt werden müssen.

So kommt Heck schließlich zu dem Resultate, daß es unzulässig sei, aus § 13 der Hunsegauer Küren die Existenz der ständischen Bußabstufung zu folgern!

Einer Kritik dieser Leistung will ich mich enthalten.

Hecks Ansehen als Forscher hätte weniger gelitten, wenn er einfach erklärt hätte, $\mathrm{da} B$ er die Unhaltbarkeit seiner bisherigen Erklärungsversuche einsehe und daher einräume, daß im Jahre 1252 im Hunsegau über dem Gemeinfreien der Edeling gestanden und $\$ 13$ der Hunsegauer Küren keinen anderen Zweck habe als das alte Rechtsverhältnis zwischen den beiden Geburtsständen der Edelinge und der Gemeinfreien zu konserviren.

Wie Heck, als er sich dem klaren Wortlaute der Hunsegauer Edelingsstelle gegenüber keinen Rat mehr wußte und doch um jeden Preis recht behalten wollte, kurzerhand zu dem verzweifelten Mittel griff, der Stelle den Charakter einer allgemeinen, für zukünftige Geschehnisse berechneten gesetzlichen Bestimmung abzusprechen und die von ihr verwendeten Gattungsbegriffe durch Individualbegriffe zu ersetzen, so hat man neuerdings einer Stelle der Hunsegauer Bußtaxen den Charakter einer gesetzlichen Bußtaxe absprechen und den Satz auf eine bestimmte Persönlichkeit beziehen wollen. Nach Siebs soll nämlich $\S 47$ der Hunsegauer Bußtaxen (Abba sîn hôd of erâwad thribête ti bêtane,

1) Nach dieser Auslassung scheint es ja fast, als ob Heck an unsrer Stelle thene etheleng und ebenso thene mon als Plural aufgefaßt hätte!! Dies wäre ein neuer hübscher Beleg für seine Beherrschung des Altfriesischen. 
allerec bi fiarda tuêde scill. Nû ist al gôd, nû heth abba sinne hôd; thâcherem nember nerthe, thâch scelt al gôd wertha), und zwar nicht nur die Verse, sondern auch die eigentliche Bußtaxe, als ein platter Schreiberscherz betrachtet und die ganze Stelle auf eine bestimmte Persönlichkeit des Namens $A b b a$ bezogen werden. ${ }^{1}$ ) Aber was Siebs dafür geltend macht, ist nicht durchschlagend. Heck hat daher erwogen, ob man nicht doch in jenem $a b b a$ einen abbas "Abt" wiederfinden könne. ${ }^{2}$ Freilich gab es in der Zeit, welcher die Hunsegauer Bußtaxen angehören, im Hunsegau noch kein Kloster, denn zu Klostergründungen kam es daselbst erst seit der zweiten Hälfte des 12. Jahrhunderts. Indes Heck weiß sich zu helfen. Er behauptet kurzweg, daß die Hunsegauer Bußtaxen „in der Masse ihrer Bußzahlen frühestens in das 12. Jahrhundert und zwar eher in die zweite Hälfte als in die erste, möglicherweise aber auch in die erste Hälfte des 13. gehören ". ${ }^{3}$ ) Diese Bußzahlen seien eine Fortbildung derjenigen Zahlen, die in den Allgemeinen Bußtaxen vorkommen. Die Fortbildung habe sich durch eine doppelte Umrechnung vollzogen, eine Umrechnung nach dem Verhältnisse von 6:11 und eine Verdoppelung. Eine Nachricht von diesen Vorgängen sei uns in den beiden ersten Wergelderhöhungen (auf 100 Pfund und auf 200 Pfund) erhalten, die im Fivelg. Ldr. überliefert seien. Die Abbanotiz gehöre, wie die Teilbarkeit der Zahl durch 11 beweise, derselben Zeit an, wie die Masse der Bußzahlen. ${ }^{4}$ ) Hier liegt wieder eines von den Heckschen "Versehen" vor. Es ist ihm nämlich das Unglück passiert, in dem zweiten Wergeldbetrage des Fivelg. Ldr., der seine Behauptung beweisen soll, das Wort "Mark" der Quelle mit den Buchstaben zu schreiben, mit welchen man sonst das Wort "Pfund" zu schreiben pflegt. An jener Stelle des Fivelg. Ldr. ${ }^{5}$ ) wird berichtet, daß man einst das "Geld" von ",e punda" auf „ec merka" erhöht habe. Die Stelle rechnet nach der Mark und dem Pfunde Grenslachta und setzt das Pfund Grenslachta mit

1) Vgl. Siebs bei Heck in den Mitteilungen des Instituts für österreichische Geschichtsforschung VII S. 771-774. - ${ }^{2}$ ) A. a. O. S. $770 \mathrm{f}$. - 3) A. a. O. S. 769. - 4) A. a. O. S. 769 Anm. 4. - 5) Hettema Fiv. Ldrgt. S. 132. 
1/15 Mark Sterling gleich. Die von ihr verwendete Mark $\left(=160\right.$ Groninger Kleine) betrug also $\left({ }^{1 / 15} \times{ }^{2} / 3=\right)^{2}{ }_{1+5}$ Mark Sterl. und jene $c$ munda (=120 Pfund) machten $(120 \times 1 / 15=)$ 8 Mark Sterl. und jene cc merk $(=2 \times 120$ Mark) genau $\left(240 \times{ }^{2 / 45} \Rightarrow 10^{2 / 3}\right.$ Mark Sterl. aus. Das neue "Geld" betrug also nicht das Doppelte, sondern nur ${ }^{4 / 3}$ vom älteren "Gelde". 8 Mark Sterlinge enthielten, wie wir schon gezeigt haben ${ }^{1}$ ), 5 röm. Pfund reinen Silbers. Sie waren demnach mit $5 \times 264$ oder $51 / 2 \times 240$ Pippinischen Denaren gleichwertig, d.h. jene $c$ punda waren derselbe Betrag, den Lex Fris. XV dem homo liber gibt: Compositio liberi litrae $V$ et dimidia per veteres dencorios. In Rednathpfennigen berechnet ergab diese Summe genau 30 Geldmark, also die Hälfte des jechtigen Geldes der Rüstringer Bußtaxen! Die cc merka aber, auf welche dieser Wergeldbetrag nach jener Fivelgauer Angabe erhöht wurde, machten in Rednathpfennigen genau 40 Geldmark, d. i. das Wergeld der jüngsten Bestandteile der Allgemeinen Bußtaxen aus, das den $53^{1 / 3}$ altfriesischen Goldschillingen an Wert gleich war, auf die sich das ostfriesische Freiengeld unmittelbar nach der großen Wergeldreduktion des 8. Jahrhunderts belaufen hatte. Durch jene Fivelgauer Angabe wird uns also die Tatsache verbürgt, daß in Ostfriesland das 778 durch Karl d. Gr. festgesetzte Freienwergeld ( $5^{1 / 2}$ Pfund zu je 240 alten karol. Denaren) im Mittelalter wieder auf den alten Betrag ( $\left(53^{1}{ }_{3}\right.$ fries. Goldschill. $=$ 40 Geldmark) erhöht worden ist. ${ }^{2}$ )

Hecks Versuch, die durch 11 teilbaren Bußzahlen der Hunsegauer Bußtaxen seinerseits zu erklären und meine Behauptung, daß es sich um Pippinische Münze handele, zu widerlegen, ist, wie man sieht, völlig gescheitert. Es ist schade, daß er die Münzverhältnisse der reichhaltigen und interessanten Hunsegauer Bußtaxen noch nicht verstehen gelernt hat. Er würde sonst wohl erkannt haben, daß der

1) Vgl. oben S. 60 f. $\left.-{ }^{2}\right)$ Die sämtlichen Beweise für diese Tatsache wird meine Geschichte des friesischen Geldwesens bringen. Wenn Heck (Ständeverbältnisse S. 93) hier von einer bloßen ${ }_{n}$ numismatischen Hypothese" spricht, so zeigt er damit von neuem, daß er von den Veränderungen, welche die friesischen Wergelder im Laufe des Mittelalters erfahren haben, gar nichts ahnt. 
Grundstock dieser wichtigen Aufzeichnung noch nach altfriesischem und nach Pippinischem Gelde rechnet. ${ }^{1}$ ) Nach dem letzteren rechnet aber auch die Abbastelle! Wie wäre ein Mönch des 12. oder 13. Jahrhunderts darauf verfallen, einen Geldbetrag in dieser Münzsorte auszudrücken!2)

\section{§ 4. Der Szêremon.}

Obwohl ich bei der Besprechung des friesischen szêremon den Schluß des $\$ 45$ der Hunsegauer Bußtaxen ausdrücklich als lückenhaft bezeichnet habe ${ }^{3}$ ), sind Heck und His auf den unglücklichen Gedanken gekommen, diese Szêremon-Stelle als ein völlig unversehrtes, widerspruchsfreies Zeugnis hinzunehmen. Sie haben gar nicht bemerkt, daß die Stelle in der Fassung, in welcher sie in den beiden Hunsegauer Handschriften überliefert ist, einen handgreiflichen Unsinn aussagt. So lesen sie denn aus ihr heraus, daß die Frau "ganz generell $1^{1 / 2}$ mal so hohe Bußen als der Szeremon" gehabt habe, und machen sie zum Hauptstützpunkte einer Polemik gegen meine Ausführungen über den Stand der Szêremen. ${ }^{4}$ ) Es ist nicht eben schwer, die Grundlosigkeit dieser Polemik nachzuweisen.

1) Vgl. hierzu Zeitschr. XXVII S.117. Die sehr verschiedenen Geldsorten, nach welchen in den Hunsegauer Bußtaxen gerechnet wird, sollen in meiner Geschichte des friesischen Geldwesens besprochen werden. - ${ }^{2}$ ) Erst wenn die Zeit, aus der $\S 47$ der Hunsegauer Bußtaxen stammt, festgestellt sein wird, werden die syntaktischen Eigentümlichkeiten der Stelle zu betrachten sein. Heck stellt (Mitt. d. Inst. f. österr. Geschichtsf. VII S. 768) bei der Besprechung der Stelle die Sache so dar, als ob ich ,der ersten Autorität auf dem Gebiete der friesischen Sprache, Siebs", nicht gerecht gewordeu wäre. Ich möchte nur wissen, inwiefern. Übrigens pflege ich auch ,Autoritäten“ gegenüber auf die eigene Uberzeugung nicht zu verzichten! Heck beschäftigt sich seit einiger Zeit eifrig mit der Autoritätenfrage, wie er ja speziell nach rechtsgeschichtlichen Autoritäten sucht, welche bereit wären, ihn auf dem Gebiete der friesischen Rechtsgeschichte als Autorität zu proklamieren. Ich wärde auch nach einer solchen Proklamation an meiner Überzeugung festhalten, daß er von der friesischen Numismatik und Münzmetrologie nichts Gründliches weiß und daß seine Ständetheorie eine schwere Verirrung ist, zu der er durch seinen Glauben, das friesische Altertum genau zu kennen, verleitet worden ist. 3) Zeitschrift XXVII S. 310. - - 4) Heck a. a. O. S. $74 \mathrm{ff}$., His in dieser Zeitschrift XXVIII s. $442 \mathrm{f}$. 
Heck erhebt gegen mich den Vorwurf, daß ich von den vier Zeugnissen über den Szêremon nur die beiden Brokmer beachtet, „die signifikanteren Stellen aus Hunsingo aber in der Darstellung und deshalb wohl auch bei der Würdigung beiseite geschoben hätte. Auch die so zahlreichen Kontrollnachrichten über die Stellung der Frau seien mir unbekannt geblieben oder nicht berücksichtigt worden, da sie mit meiner Auffassung der Frauenbuße nicht vereinbar wären". 1) Ein sonderbarer Vorwurf! Seit wann hat denn der Verfasser einer wissenschaftlichen Monographie die Pflicht, über Dinge zu sprechen, welche gar nicht zum Thema gehören? Das Thema meines Aufsatzes lautete doch dahin, "genau festzustellen, was die friesischen Rechtsquellen des 11.--13. Jahrhunderts in Wirklichkeit über die friesischen Standesverhältnisse aussagen ". ${ }^{2}$ ) Eine Erörterung der Frauenbuße hatte ich mir nicht zur Aufgabe gemacht. Entsprechend habe ich von den vier Szêremon-Stellen die beiden, welche für das Ständeproblem in Betracht kommen, in den Fordergrund gestellt und auf die beiden anderen, die rom Sonderfrieden der Frau handeln und für die Erkenntnis des friesischen Ständewesens keine unmittelbare Bedeutung haben, nur der Vollständigkeit wegen kurz hingewiesen. ${ }^{3}$ ) Freilich, hätte ich ahnen können, daß man die eine dieser beiden Stellen (Hunsegauer Küren $\S 45$ ) so kritiklos interpretieren würde, wie es nunmehr geschehen ist, so würde ich mich auf den Hinweis, daß der Schluß dieser Stelle lückenhaft sei, nicht beschränkt, sondern diesen Schluß eingehend erläutert haben.

Wenn ich hier, um auch den letzten Zweifel, daß Heck und His für eine verlorene Sache streiten, zu zerstreuen, die vier Sżêremon-Stellen noch einmal auf ihre Bedeutung für die Ständekontroverse prüfe, so soll dabei alles, was nicht unbedingt zur Sache gehört, beiseite bleiben. Heck hat die beiden Brokmer Stellen, denen entscheidende Bedeutung für das Ständeproblem zukommt, nur obenhin gestreift ${ }^{4}$ ), die beiden Hunsegauer Stellen dagegen, die für dieses Problem nur mittelbare Bedeutung haben, ausführlich besprochen.

1) A. a. O. S. $\left.85 .-{ }^{2}\right)$ Zeitschrift XXVII S. $275 .-{ }^{3}$ ) A. a. O. S. $308 \mathrm{ff}$. 4) A. a. O. S. $85 \mathrm{f}$. 
Eine methodische Untersuchung der ostfriesischen Standesverhältnisse hat natürlich gerade umgekehrt zu verfahren.

Nach den beiden Brokmer Stellen ${ }^{1}$ ) soll bei Frauenraub und dann, wenn ein minderjähriges Mädchen hingegeben oder weggelockt wird, ên tuêde szêrmonnis ield als Privatbuße und ein halb so hoher Betrag als Friedensgeld, bei Notzucht dagegen ên szêrmonnis ield als Buße und ein halb so hoher Betrag als Friedensgeld gezahlt werden.

Die Brokmer gehörten zu der östlichsten friesischen Gruppe, die das Land zwischen der ostfriesischen Bruch- und Hochmoorstrecke und der unteren Weser innehatte. Rüstringen und Brokmerland sind die einzigen Landschaften dieses Gebietes, aus welchen sich umfängliche Rechtsaufzeichnungen erhalten haben. Zur Erläuterung der Brokmer Rechtsquellen sind daher in erster Linie die Rüstringer Rechtsquellen heranzuziehen und umgekehrt. Wenn Heck, um seine Lehre von der Standeseinheit, die im Mittelalter bei den Friesen geherrscht habe, zu retten, sich zu der Behauptung versteigt, daß man die Rüstringer Rechtsverhältnisse nicht zur Aufhellung der Brokmer Rechtszustände verwenden dürfe ${ }^{2}$ ), so beweist er damit nur, daß er sich noch nicht über die Herausbildung der "terra Brocmanniae" unterrichtet hat; und dasselbe geht aus seiner schiefen Behauptung hervor, daß, das Brokmer Recht nur ein Zweig des Emsiger Rechtes", das Emsiger Recht das "Mutterrecht" des Brokmer Rechtes sei. ${ }^{3}$ )

Die Rüstringer Küren, welche nach einem jechtigen Gelde von 40 Mark rechnen, setzen die Privatbuße für Notzucht auf 20 Mark, also auf ein halbes Normalgeld fest. ${ }^{4}$ ) Genau dasselbe wird durch eine Satzung bestimmt, welche nur in der Handschrift von 1327 erhalten ist. ${ }^{5}$ ) Wenn also der Brokmerbrief diese Buße auf ên szêrmonnis icld normiert, so führt dies auf die Gleichung: 1 szêrmonnis ield $=$ $1 / 2$ Normalgeld.

Dieselbe Gleichung erhält man, wenn man die Brokmer und Rüstringer Angaben über das Friedensgeld, welches der

1) Brokmerbrief $\S 106 \mathrm{f}$. (Fries. Rq. 166). Den genauen Text hat Siebs bei Heck a. a. O. S. 76 f. gegeben und eine Übersetzung beigefügt. ${ }^{2}{ }^{2}$ S. 85 f. - ${ }^{3}$ ) S. $\left.82 f .-{ }^{4}\right)$ Vgl. die 9. Rüstringer Küre Fries. Rq. 116. - $\left.{ }^{5}\right)$ Fries. Rq. $542 \$ 51$. 
Notzüchter zu zahlen hatte, vergleicht. Nach den beiden angeführten Rüstringer Stellen stand auf Notzucht ein Friedensgeld von hundred merlia (120 Mark). Genau ebensoviel, nämlich hundred merkia, betrug in Rüstringen der normale Totschlagsfredus. ${ }^{1}$ ) Entsprechend setzt eine alte Rüstringer Satzung ${ }^{2}$ ) auf Notzucht genau dasselbe Friedensgeld wie auf Totschlag, nämlich 30 Volle Mark (=120 Reilmark).

Wie in Rüstringen auf Notzucht und auf Totschlag genau dasselbe Friedensgeld stand, so auch im Brokmerlande. Das Victorburer Strafregister ${ }^{3}$ ), das aus der zweiten Hälfte des 13. Jahrhunderts, also aus derselben Zeit wie der Brokmerbrief stammt, bemißt die Strafe umbe tha necthest ebenso wie die Strafe pro homicidio auf 5 Mark, und der jüngere BrokmerEmsiger Rechtsvertrag, der ebenfalls der zweiten Hälfte des 13. Jahrhunderts angehört, bestimmt ausdrücklich: Iudices accipiant a sanguinis effusore duas marcas sterlingorum, und hinsichtlich des Notzüchters: Iutlices pro frethebannis duas marcas sterlingorum accipiant. ${ }^{4}$ )

1) Vgl. das Friedensgeld zu hundred merka in den älteren Rüstringer Küren 1, 2, 4, 7, 8, 9, 11, 12, 14, in der neveren Küre 12 und in dem Texte von $1327 \S \S 45,46,51,53,56$, endlich im Rüstringer Sendrecht Fries. Rq. 128, 23 und 13. - $\left.{ }^{2}\right)$ Fries. Rq. 123, 23. - ${ }^{3}$ ) His, Strafrecht der Friesen S. 360 ft. - *) Fries. Rq. 136. Die Brokmer und Rüstringer Rechtsquellen stehen mit ibrer Bestimmung, daß bei Notzucht dasselbe Friedensgeld wie bei einem Totschlage zu zahlen sei, keineswegs allein da. Auch in Mittelfriesland bestand eine solche Vorschrift zu Recht. Als Totschlagsfredus waren hier, wie zahlreiche Stellen (Fries. Rq. 411, 37. 412, 24. 474 §§ 1, 2, 3, 5. 384, 12. 560. 386, 35. 387, 6. $446,19)$ ergeben, au die "Herren" und "Leute" 80 Pfund zu zahlen; und bei Notzucht sollte nach $\S 30$ des Schulzenrechts der Verbrecher tachtich pond herem ende lyodem iaen (Fries. Rq. 391, 8)! Es handelt sich bei dieser Gleichbeit der Totschlags- und der Notzuchtstrafe um eine alte gemeinfriesische Rechtsnorm. Nach Add. leg. Fris. III 76 sollte, wenn ein Freier die Gattin eines andern geraubt hatte, der Verbrecher seinen veregildus als Friedensgeld an den König zahlen. Der Totschlagsfredus aber betrug wach Lex XIV 1-2 und X in Mittelfriesland, nach XIV 3 in Westfriesland und nach VII 2 auch in Ostfriesland einen weregildus! Man hat dann auf jede Frauenvergewaltigung, mochte die Verletzte eine Jungfrau oder Witwe oder die Gattin eines andern sein, dieselbe Strafe gesetzt. Vor der friesischen Wergeld- und Bußenreduktion, die in der zweiten Hälfte des 8. Jahrhunderts stattfand, belief sich diese Strafe auf einen älteren weregildus, der das Vierfache des 
Diesen positiven Zeugnissen gegenüber weiß sich Heck keinen anderen Rat, als meinen Hinweis auf die Rüstringer Notzuchtstrafe kurzerhand eine unverwertbare Parallelbeobachtung zu nennen und sich über die Brokmer Quellenzeugnisse auszuschweigen! Die Beweislage muß ihm nicht ganz geheuer vorgekommen sein. Sonst wäre er über sie nicht mit der allgemeinen Wendung, daß „die Notzuchtbuße jedenfalls in späterer Zeit eine lokal verschiedene gewesen sei“, und mit einem Hinweise auf seine Rezension von His' Strafrecht hinweggeschlüpft. Was er an der zitierten Stelle ${ }^{1}$ ) über die lokal verschieden bemessene Notzuchtbuße beibringt, gehört gar nicht hierher.

Es steht also quellenmäßig fest, daß nach dem Rechte der Brokmer wie nach dem altfriesischen Rechte überhaupt der Frauenvergewaltiger dasselbe Friedensgeld verwirkte wie der Totschläger. Dieses Ergebnis läßt sich durch keine Interpretationskünste beseitigen. Wemn also bei den Brokmern der Notzüchter ein halbes szêremonnis-icld als Friedensgeld zu zahlen hatte, so folgt daraus unmittelbar, daß sich im Brokmerlande auch der normale Totschlagsfredus auf ein halbes szêremonnis-ield belaufen hat. Es muB sich also aus dem Verhältnisse, welches in Ostfriesland zwischen dem Normalgelde und dem normalen Totschlagsfredus obwaltete, mit voller Sicherheit ergeben, wie der Szêremon zu dem

jüngeren ausmachte. Daraus, daß die Reduktion nicht überall gleichmäßig durchdrang, ist es zu erklären, daß sich hier und da die Strafe für Frauenvergewaltigung auf der alten Höhe hielt, d. h. viermal soviel wie der spätere Totschlagsfredus ausmachte. Dies war z. B. in Langewold der Fall, wie sich aus den älteren und jüngeren Langewolder Kürev klar ergibt. So ist auch die hohe Strafe zu erklären, welche in der 15. friesischen Küre auf Notzucht gesetzt ist. Diese Strafe ist hier auf 12 große Mark, d. h. auf 180 Schillinge zu je 12 altfries. Silberpfennigen bemessen (vgl. Zeitschr.XXVII $293 \mathrm{ff}$.), wovon aber an den Schulzen 14 kölnische oder 20 friesische Schillinge zu fallen hatten. Als eigentliches Friedensgeld blieben also 160 Schillinge zu je 12 fries. Silberpfennigen oder $531 / 3$ altfries. Goldschill., d.h. der ältere Freienweregildus, übrig.

1) Gött. Gel. Anz. 1902 S. 865. Heck (S. 60) bezeichnet diese Rezension als seine auf dem Gebiete meiner Arbeit neueste und wichtigste Publikation. Leider babe ich aus dieser wichtigsten Publikation nichts erfahren, was neu und zugleich richtig wäre. 
freien Manne, für den die friesischen Rechtsquellen berechnet sind, gestanden hat.

Der Totschlagsfredus kam nach altostfriesischem Rechte dem vierten Teile des "Geldes" gleich. Ich habe dies bereits in meinem Aufsatze "Weregildus und simpla compositio" gezeigt. $\left.{ }^{1}\right) \mathrm{Lm}$ mich nicht zu wiederholen, will ich die daselbst beigebrachten Stellen der friesischen Rechtsquellen hier nicht von neuem besprechen, auch nicht die sämtlichen sonstigen Beweise für meine Behauptung aufführen ${ }^{2}$ ), sondern mich auf eine Erörterung der Argumente beschränken, welche His und Heck gegen meine Ansicht rorgebracht haben.

His macht zunächst geltend ${ }^{3}$ ), daß nach $\$ 10$ der Hunsegauer Küren der Totschlagsfredus ungefähr ein Drittel des Wergeldes betragen habe. Dieser Einwand beruht lediglich darauf, daß His die Worte ,umbe allerck dâddela" in diesem Paragraphen nicht richtig aufgefaßt hat, und ist durch meine frühere Ausführung ${ }^{4}$ ) bereits entkräftet. Sodann führt His an, daß sich nach dem Vertrage zwischen dem Fivelingalond und dem Oldamte (Fries. Rq. 295) der Totschlagsfredus etwa auf ein Fünftel, nach dem Sielrecht der drei Delfsiele von 1317 (Fries. Rq. 288) auf genau ein Fünftel, nach den Humsterländer Küren (Fries. Rq. 355) etwa auf ein Siebentel des Wergeldes belaufen habe. Aber wer sich diese Quellen des 14. Jahrhunderts genauer ansieht, wird bald erkennen, daß ihre Angaben für die Entscheidung der Frage, wie sich nach altostfriesischem Rechte der normale Totschlagsfredus zum Normalgelde verhalten hat, ohne Bedeutung sind.

Das Sielrecht (Rq. 288) erwähnt den den Leuten zustehenden Friedensgeldbetrag überhaupt nicht mehr, sondern spricht nur von dem brekma, den die Richter erhalten sollten, und zwar wird ein brckma von 12 Mark Sterlingen in $\S 1$ einer Compositio von 60 , in $\S 7$ dagegen einer Compositio von 40 Mark Sterlingen parallel gestellt. Daß wir es hier nicht mit einer Bestimmung des gemeinen Fivelgauer Landrechts zu tun haben, ersieht man aus den Sander Deich-

1) Vgl. Zeitschrift XXVIII S. 138-141 und meine Bemerkungen in Zeitschrift XXVII S. 280, 300-302, 310. - 2) Die Gesamtheit der Belege soli meine Geschichte des friesischen Geldwesens bringen. 3) Zeitschrift XXVIII S. 442. - ") Zeitschrift XXVIII S. 140. 
satzungen ( $\mathrm{Rq.} 290 \mathrm{f}$ ), die demselben Jahre angehören. Sie stellen einer Compositio von 60 Mark Sterl. einen brekma von 6 und der Compositio von 40 Mark Sterl. einen brekme von 4 Mark Sterl. gegenüber! Die alte Fivelgauer Friedensgeldordnung läßt sich aus diesen für ganz besondere Verhältnisse berechneten Bestimmungen über den brekma der Sielrichter nicht ersehen. ${ }^{1}$ )

Daß man die alte Friedensgeldordnung des Gaues Hugmerke nicht aus den ganz jungen, mangelhaft überlieferten Küren von Humsterland ${ }^{2}$ ) ohne Berücksichtigung der Langewolder und Fredewolder Rechtsquellen feststellen kann, sollte man eigentlich nicht erst zu sagen brauchen. Die Humsterländer Küren zeigen nur, daß es auch in der Hugmerke im 14. Jahrhundert mit der alten Friedensgeldordnung vorbei war. Nach $\S 1$ soll bei einem unqualifizierten Totschlage das "Geld“ 8 Mark Englisch, also 96 Schill. Englisch, betragen und den Richtern ein Friedensgeld von 14 Schill. Englisch gezahlt werden. Nach $\S 6$ sollten bei Hausbrand Buße und Friedensgeld ebensoviel, bei nächtlicher Brandstiftung dagegen dieBuße 16 Mark Englisch und das Friedensgeld 24 (!) Schill. Englisch betragen. Hier liegt ein Fehler zutage. In $\$ 7$ ist auf das Abschlagen eines Hauptgliedes ein Friedensgeld von 7 Schill. gesetzt, während als Friedensgelder für die drei Lähmungsgrade 6, 4 und 2 Schill. gefordert werden. Dies sind ganz junge Verhältnisse, denn auch in der Hugmerke wurde einst das völlig gelähmte Glied halb so hoch wie das völlig abgeschlagene und das Hauptglied halb so hoch wie das Leben gebüßt. ${ }^{3}$ ) Wenn also auf die höchste Lähmung eines Hauptgliedes ein Friedensgeld von 6 Schill. stand, so

1) Im 14. Jahrhundert befindet sich die alte friesische Friedensgeldordnung bereits in voller Auflösung. Der alte liudafrethe wird hier und da gauz beseitigt, anderwärts zur Komposition oder zum richterlichen Brekma geschlagen. Wenn die Fivelgauer Sielrechte, die die Compositio augenscheinlich verdreifachten, die dreifache Compo. sitio zu 60 Mark Sterl. ansetzen, während das "Geld" im Fivelgau in der zweiten Hälfte des 13. Jahrhunderts auf 16 Mark Sterl. festgesetzt worden war, so hatten sie wohl den Liudafrethe mit zur Komposition gezogen. Vgl. auch den Schluß der Fivelgauer Aufzeichnung über die Erhöhung des Geldes bei Hettema, Fivelg. Landregt S. 132. 2) Fries. Rq. $\left.350 \mathrm{ff},-{ }^{3}\right)$ Vgl. z. B. Fries. Rq. $367 \S \S 10$ und 11. 
muß das Friedensgeld beim Abschlagen eines Hauptgliedes einst 12 und der Totschlagsfredus 24 Schill, also 2 Mark Englisch, betragen haben. Der Totschlagsfredus (2 Mark Englisch) hat sich also einst auch im Humsterland, gerade so wie in Langewold und in Fredewold, zum Normalgelde (8 Mark Englisch) wie 1:4 verhalten.

Auch die zwischen dem Fivelingalond und dem Fivelgauer Oldamt vereinbarten Küren (Fries. Rq. $298 \mathrm{ff}$.) lassen sich für die Feststellung der altostfriesischen Friedensordnung nicht verwerten, weil wir nicht wissen, wie hoch sich zur Zeit ihrer Vereinbarung das Normalgeld im Oldamt belief. Nach $\S 1$ sollte bei einem Totschlage zwischen den beiden Ländern das "Geld" 16 Mark Englisch betragen und an die Richter eine Hauptlöse im Betrage von 26 Schill. Englisch fallen. Statt XXVI, wie in dem von Richthofen abgedruckten jungen niederdeutschen Manuskripte steht, liest man in der Fivelgauer Rechtshandschrift ${ }^{1}$ ) XXXVI! Nach $\$ 5$ sollte beim Abschlagen eines Hauptgliedes die Buße 8 engl. Mark, also die Hälfte des vereinbarten "Geldes" betragen und den Richtern 1 Mark, nach $\S 6$ bei höchster Lähmung $1 / 2$ Mark gezahlt werden. Dies führt auf einen Totschlagsfredus von 2 Mark, also von 24 Schill. Englisch, während der Totschlagss. fredus des Fivelingalondes damals 4 Mark Englisch betrug. Wahrscheinlich beruht also jener Totschlagsfredus von 36 Schill. Englisch auf einem Kompromiß. Der Fivelingoer Totschlagsfredus machte 4 Mark aus, und der des Oldamtes betrug wohl 2 Mark. So mochte man vereinbart haben, daß bei einem Totschlage zwischen den beiden Ländern der Totschlagsfredus drei Mark betragen solle.

Die Höhe des Oldamter Normalgeldes ist nicht bekannt. Falls es sich wie das der Hugmerker auf 8 Mark Englisch belaufen haben sollte, würde es sich zu dem Friedensgelde von 2 Mark Englisch wie 4:1 verhalten haben! Möglicherweise ist aber das Oldamter "Geld" damals bereits höher gewesen, das alte Friedensgeld aber noch bestehen geblieben. Sicherheit läßt sich hierüber aus den uns zur Verfügung stehenden Quellen nicht gewinnen!

1) Hettema S. 146. 
Nach allem erweist sich von den Einwänden, welche His gegen mein Ergebnis erhoben hat, daß nach altostfriesischem Rechte der normale Totschlagsfredus zum Normalgelde wie 1:4 stand, bei näherem Zusehen keiner als stichhaltig. Aus den älteren ostfriesischen Rechtsquellen das Verhältnis zwischen Privatbuße und Friedensgeld zu ermitteln, was die Vorbedingung für eine gründliche Bekämpfung meiner Ansicht wäre, hat His gar nicht erst versucht.

Was wendet nun Heck gegen mein Ergebnis ein? Er bezeichnet es als "sicher falsch", denn der Totschlagsfredus habe ursprünglich in Ostfriesland nicht $1 / 4$, sondern $3 / 10$ des Wergeldes betragen. Er verweist deswegen auf den Anhang seiner Abhandlung (S. $222 \mathrm{ff}$.: „Das hohe Friedensgeld in Rüstringen") und auf seine Ausführungen in den Göttinger Gel. Anz. S. $863 \mathrm{ff}$. Was an der letzteren Stelle zu lesen ist, findet sich auch in jenem Anhange. Es genügt also, diesen Anhang zu durchmustern, dessen „Erörterungen es ganz zweifellos erseheinen lassen, $\mathrm{da}$ (as hohe Friedensgeld in Rüstringen nach allen Nachrichten ein und denselben Wert repräsentiert, der in Geldmark 12 Geldmark betrug und sich deshalb zu dem Wergelde von 40 Geldmark, das gleichzeitig galt, wie $3: 10$ verhielt und nicht wie $1: 4^{\text {". }}$.

Ich hatte in Hecks überraschender Mitteilung, daß ihm im Rüstringer Recht ein Friedensgeld von 12 Geldmark begegnet sei, die Angabe vermißt, an welcher Stelle er diese Begegnung gehabt habe, und die Vermutung ausgesprochen, daß er ein Friedensgeld von solcher Höhe lediglich erschlossen habe. ${ }^{1}$ ) Darauf erwidert er (S. 223), daß die wichtigen Hinweise, die er in seiner Rezension von His (S. 867, 872f.) gegeben hätte, von mir übersehen worden seien. Man sollte also meinen, daß in dieser Rezension die Stelle, an der sich jene Begegnung vollzogen hat, angegeben sei. Aber alles Suchen würde vergeblich sein. Die Stelle ist auch dort nicht vermerkt. Heck hat also wirklich die Kühnheit gehabt, eine bloße Ausgeburt seiner münzmetrologischen Spekulationen für eine quellenmäßig begründete Tatsache auszugeben!

In den Rüstringer Rechtsaufzeichnungen wird der normale Totschlagsfredus in verschiedenen Geldsorten berechnet.

1) Zeitschrift XXVII S. $296 \mathrm{f}$. 
Gewöhnlich wird er zu hundred merka, d. h. zu 120 Reilmark, angegeben. ${ }^{1}$ ) Nach einer Angabe betrug er 30 Volle Mark. ${ }^{2}$ ) Endlich läßt sich aus einigen Stellen noch entnehmen, wie viel er in Geldmarken und in Schillinggewichten Gold ausgemacht hat. Heck ist $\mathrm{zu}$ dem Resultate gelangt, daß sich der normale rüstringische Totschlagsfredus auf 12 Schillinggewichte Gold oder 12 Geldmark belaufen, also zum jechtigen Gelde (40 Geldmark) wie 3:10 gestanden habe, während nach meinem Ergebnis dieser Fredus nur 10 Geldmark betrug, sich also zum jechtigen Gelde wie 1:4 verhielt.

Die Fehler, welche Heck bei seiner Berechnung untergelaufen sind, lassen sich leicht aufdecken.

Nach der Rüstringer Friedensgeldordnung, welche in beiden Handschriften den Schluß der Rüstringer Bußtaxen bildet ${ }^{3}$ ), sollte der Totschlagsfredus 6 Hauptlösungen betragen. Nun wird in einer jüngeren Rüstringer Stelle die Hauptlösung eines Aldermanns auf 2 Schillinggewichte Gold berechnet.") "Deshalb ", meint Heck (S. 223), "und wegen der Identität der Geldmark mit dem Schilling Goldgewicht würde dieser Anhaltspunkt für den Totschlagsfredus genau 12 Geldmark ergeben und zwar als sicheren Maximalbetrag. Ebenso begegnet uns ein Friedensgeld von 12 Mark (grata

1) Vgl. die Nachweise oben S. 75 Anm. 1. Daß unter dem hundred der friesischen Rechtsquellen nicht das dezimale, sondern das duodezimale Hundert zu verstehen ist, hat mir Heck, wenn auch widerstrebend, zugegeben. Es sei von dem Neuen, was meine Aufsätze gebracht, das einzige, was wenigstens nicht ganz abzulehnen sei (S. 60). Ohne Rückzugsgefechte geht es natürlich nicht ab! Die Geschicklichkeit, mit welcher Heck um das einfache Zugeständnis, sich geirrt zu haben, herumzukommen und die bessere Einsicht, die ihın der Gegner beigebracht, als Frucht eigenen Nachsinnens hinzustellen (S. 226) weiß, verdient nicht geringere Bewunderung als der Eifer, mit welchem er mich zu einem Vertreter seiner Ansichten zu stempeln sucht (S. 55 f.). Schon bat er herausgefunden (S. 200f.), daß der iunerste Kern meiner Auffassung des Wortes friling die Ansicht Hecks und das Ergebuis meiner Beschäftigung mit den Frîlingsstellen eine zwar verschleierte, aber doch unverkennbare Zustimmung zu seiner Grundanschauung sei. Ich würde mich gar nicht wundern, wenn er eines Tages entdeckte, daß der innerste Kern meiner Auffassung der friesischen Standesverhältnisse nichts anderes als Hecks Ständetheorie sei! - ${ }^{2}$ ) Fries. Rq. 124, 6. - ${ }^{3)}$ Fries. Rq. 121, 11; 538 $\S 12$. - 4) Fries. Rq. 124, 5.

Zeitschrift für Rechtsgeschichte. XXX. Germ. Abt. 
merk) in einem Einzelfalle, in dem wir die große Hauptlösung erwarten dürfen." 1 )

Die vorbehaltlose Identifizierung der Geldmark mit einem Schillinggewicht Gold ist der eine, die Annahme, daß es sich bei den 12 grata merka des Einzelfalles, auf welchen Heck hinweist, aber wohlweislich nicht näher eingeht, um Geldmarken handele, der andere Fehler der Heckschen Argumentation.

$\mathrm{DaB}$ es eine Periode gegeben hat, in welcher die Rüstringer die Geldmark und das Schillinggewicht Gold gleich hoch bewerteten, steht außer Zweifel. Das Rüstringer Markenschema sagt ausdrücklich: thiu ieldmerk thiu is ên skilling wicht goldis ${ }^{2}$ ) und entsprechend ein Zusatz zu dem jüngeren Texte der Rüstringer Bußtaxen: thiu ieldmerk thin is ên skilling. ${ }^{3}$ ) Aber diese Gleichsetzung beruhte auf einem bestimmten Wertverhältnisse des Goldes zum Silber, denn das Schillinggewicht Gold war ein Goldquantum, die Geldmark dagegen eine Silber-Rechnungsmünze. Änderte sich jenes Wertverhältnis, so mußte sich auch das Wertverhältnis des Schillinggewichtes Gold zur Geldmark verschieben, so daß dann von einer Identität dieser beiden Werte nicht mehr die Rede sein konnte. Daß eine solche Verschiebung in Rüstringen innerhalb der von den erhaltenen Rechtsaufzeichnungen umfaßten Zeit stattgefunden hat, läßt sich aus den Zusätzen zu den Rüstringer Bußtaxen deutlich ersehen. Es rechnet nämlich die nachträglich zu dem alten Bußtaxentexte hinzugesetzte Bemerkung über die Magenbuße

$2(2 \times 9$ skill. cona +2 skill. wicht goldis $)=5$ skill. $w . g$.

$$
-4 \text { skill. cona. }
$$

Daraus ergibt sich die Gleichung: 1 skilling wicht goldis = 40 skillinga cona. ${ }^{4}$ ) Dagegen werden in den Zusätzen des

1) Fries. Rq. 123, 10. - 2) Fries. Rq. 125, 4; $540 \S 27 .{ }^{3}$ ) Fries. Rq. $\left.536,17 .{ }^{4}\right)$ Die schon in der Berl.Zeitschr. für Numism. XII S. 148 f, besprochene Stelle lautet: ${ }_{n} \mathrm{Ac}$ heth hit inur ebitin, forth inur thene maga, twia niugun skillinga cona and tuira skillinga wicht goldis, thi utgong alsa felo; sa is thi maga al semin fif skillinga wicht goldis al buta fuwer skillingon cona ${ }^{\mu}$ Fries. Rq. 120, 14-19. 537, 23-27. Der Satz steht in dem Texte von 1327 an einer etwas späteren Stelle, so daß es offenbar ist, daß man diese Bemerkung über den Magen erst nachträglich als Randbemerkung zum urs prünglichen Bußtaxentexte hinzugesetzt 
Textes von 1327 einerseits 36 skill. cona mit 3 Lot, andrerseits 15 panninga wicht goldis mit 5 Lot, $71 / 2$ pann. w. g. mit $2^{1 / 2}$ Lot, 2 skill.w.g. - 11/2 pann.w. g.mit $7^{1 / 2}$ Lot geglichen. ${ }^{1}$ ) Diese vier Reduktionen führen auf die Gleichung: 1 skill.w.g. $=48$ skill. cona.

Man hat also in der Zeit, wo die Rüstringer Bußtaxen mit Zusätzen versehen wurden, bereits geschwankt, ob man das Schillinggewicht Gold mit 48 oder nur noch mit 40 Schillingen cona gleichzusetzen habe, was seinen Grund darin hatte, daß damals das Wertrerhältnis der beiden Edelmetalle von 12 auf 10 herabging. Solange Gold zu Silber wie 12:1 stand, galt das rüstringische Schillinggewicht Gold 48, seitdem aber Gold zu Silber wie 10:1 stand, nur noch 40 Schillinge cona. Die Interpolationen des Textes von 1327, die das Schillinggewicht Gold mit 48 Schillingen cona gleichsetzen, also das Gold noch auf das Zwölffache des Silbers veranschlagen, erklären ausdrücklich die Geldmark und das Schillinggewicht Gold für gleiche Werte. Daraus folgt also, daß die Geldmark nicht 40, sondern 48 Schillinge cona umfaßt hat ${ }^{2}$ ) und daß die Gleichwertigkeit der Geldmark mit dem Schillinggewicht Gold nur so lange gegolten hat, als das Wertverhältnis der beiden Metalle $12: 1$ betrug, während nach dem Sinken dieses Verhältnisses auf 10:1 das Schillinggewicht Gold nur mit $5 / 6$ Geldmark oder 40 Schillingen cona geglichen werden konnte.

Nach allem war es ein Fehler des Numismatikers Heck, die 12 Schillinggewichte Gold, auf welche sich nach einer Rüstringer Angabe die große Hauptlösung des Aldermanns belief, ohne weiteres mit 12 Geldmark gleichzusetzen. Er

hat. Nur so konnte es geschehen, daß sie der Schreiber des Manuskripts von 1327 an einer anderen Stelle in den Text nahm als der Schreiber der Oldenburger Handschrift. Im Wurster Texte steht sie an derselben Stelle wie im Oldenburger Texte!

1) Fries. Rq. $536 \S 4$ und $537 \S 9$. Der Ansatz ${ }_{n} 10^{1 / 2}$ pann.w. g. (Rq. 537, 16) ist, wie der Oldenburger Text zeigt, in $71 / 2$ pann. w. g. ${ }^{*}$ zu bessern. - 2) Daß die Geldmark 40 Schillinge cona umfaßt habe, wird nirgends gesagt oder angedeutet. Heck hat die Geldmark um $1 / 6$ zu niedrig angesetzt! Sie ist also nicht mit ihm zu 120 , sondern zu $(120 \times 8 / 5=) 144$ Rednathpfennigen zu rechnen. 
hätte sagen sollen, daß diese 12 Schillinggewichte Gold, je nachdem das Wertverhältnis der beiden Edelmetalle 12 oder 10 betrug, auf 12 oder 10 Geldmark zu bewerten seien und $\mathrm{daB}$ für die letztere Bewertung der Umstand spreche, daß sich jene Erwähnung der Hauptlösung des Aldermanns unter den jüngeren Stücken der Oldenburger Rechtshandschrift findet.

Wie steht es nun mit dem Einzelfall, den Heck für seine Bestimmung des Totschlagsfredus ins Feld führt? Die fragliche Stelle der Rüstringer Aufzeichnung über das friesische Recht beschäftigt sich mit der ungerechten Fesselung und setzt auf die schlimmste Art tô bôte twilif merk and twilif merk tha liodon tô fretha, al bi there grâta merk. ${ }^{1}$ ) Dieses Friedensgeld zu 12 Großen Mark soll nach Heck ebenfalls 12 Geldmark betragen, also $3 / 10$ vom Normalgelde ausmachen.

Man wird nicht verlangen dürfen, daß Heck das schwierige Gebiet der friesischen Fesselungsbußen beherrscht. Aber wenn an jener Stelle das Friedensgeld genau so hoch wie die Buße normiert wird, so würde ein sorgsamer Forscher doch wenigstens geprüft haben, wie hoch sich nach anderen ostfriesischen Quellen die höchste Fesselungsbuße belief. Er würde gefunden haben, daß sie allenthalben weit mehr als $3 / 10$ Normalgeld betragen hat. Im Rüstringer Texte der Allgemeinen Bußtaxen ist sie z. B. auf $2 / 3$ "Geld" bemessen. Mit jenen 12 Großen Mark können eben nicht Geldmarken, sondern nur wirkliche Große Marken, d.h. Marken zu 180 Pfennigen, gemeint sein. Die Partie, zu welcher unsere Stelle gehört, rechnet nach dem Rednath(Kawing)-Pfennige. 180 Stück solcher Pfennige machten 60 altfriesische Pfennige, 12 Große Mark also 60 Schillinge zu je 12 altfries. Silberpfennigen aus. Hierzu stimmt, daß in $\S 9$ der Hunsegauer Bußtaxen auf die ungerechte Fesselung der schlimmsten Art eine $\mathrm{Buße}$ von $4 \times 15$ Mark gesetzt wird ${ }^{2}$ ), denn dieser Betrag machte, da die Mark der Hunsegauer Bußtaxen 12 altfriesische Silberpfennige galt, genau 60 Schillinge zu je 12 altfriesischen Pfennigen aus. Hierzu stimmt ferner, daß sich

1) Fries. Rq. 123, 9-11. - 2) Fries. Rq. 340, 28. 
die Buße für jene Fesselungsart nach dem jüngeren BrokmerEmsiger Rechtsvertrage auf 4 Mark Sterlinge (= 48 Schillinge Sterlinge) belief. $\left.{ }^{1}\right)$ Denn der Sterling verhielt sich zum altfriesischen Pfennige genau wie 5:4. Die 48 Schillinge Sterlinge waren also mit 60 Schillingen zu je 12 altfries. Silberpfennigen gleichwertig.

Wie unsere Erörterung gezeigt hat, ließe sich die Hecksche Behauptung, daß in Rüstringen der 'Totschlagsfredus zum Normalgelde wie $3: 10$ gestanden habe, nur halten, wenn man kritiklos die Große Mark mit der Geldmark zusammenwerfen, wenn man das in den Zusätzen der Rüstringer Bußtaxen sich spiegelnde Sinken des Goldwertes vom $\mathrm{Zwölffachen} \mathrm{auf} \mathrm{das} \mathrm{Zehnfache} \mathrm{des} \mathrm{Silberwertes} \mathrm{igno-}$ rieren und wenn man sich zu der quellenwidrigen Annahme entschließen würde, daß die Geldmark nicht 48 , sondern 40 Schillinge cona umfaßt habe.

Wenn man die beiden für den rüstringischen Totschlagsfredus angegebenen Beträge von 30 Vollen Mark und 120 Reilmark mit dem rüstringischen Markenschema, das die Volle Mark zu 16, die Reilmark zu 4 Schillingen cona berechnet, kombiniert, so führt Hecks quellenwidrige Gleichsetzung der Geldmark mit 40 Schillingen cona natürlich zu einem Friedensgelde von 12 Geldmark. ${ }^{2}$ ) Setzt man aber quellengemäß die Geldmark zu 48 Schillingen cona an, so ergibt jene Rechnung für das Friedensgeld den Betrag von 10 Geldmark! Und dieses Ergebnis wird durch die älteren Rüstringer Küren bestätigt. Diese Rechtsquelle rechnet nach einem "Gelde" zu 40 Mark, worunter 40 Geldmark zu verstehen sind, und gibt den normalen Totschlagsfredus regelmäßig zu hundred merka, d. i. zu 120 Reilmark, an. Aber dort, wo sie ältere Satzungen übernimmt und dabei das verdreifachte Friedensgeld aufführt, verwendet sie die schwereren Münzsorten. So setzt die dritte Küre das Friedensgeld für Mord ${ }^{3}$ ) auf 30 Mark fest:

1) Fries. Rq. 136, 20: „Si quis violenter captus fuerit et vinctus fuerit et tanquam fur ductus fuerit et hoc sex iudices testentur quod hoc factum fuerit, quatuor marcas sterlingorum pro emenda recipiat et iudices simplices frethebannos accipiant." $-{ }^{2}$ ) Vgl. Heck S. 225 f. 3) Daß die östlichste Friesengruppe den Mord und die ihm gleich- 
Sa hwa sa enne mon slê and Wer einen Mann erschlägt thes nêna iechta ne lède, thet und dies verheimlicht, daß er hi tritich merke breke (Fries. eine Brüche von 30 Mark Rq. 115). zahle.

In der 10. Küre (Rq. 116) wird dieses verdreifachte Friedensgeld auf 30 Schillinggewichte Gold normiert. Endlich gibt die 13. Küre (Rq. 116) denselben Betrag zu 8 Mark Silber, in ihrem zweiten Teile zu 30 Mark und daneben den einfachen Fredus zu 10 Mark an. $\left.{ }^{1}\right)$ Nach diesen ausdrücklichen Angaben hat also damals der normale Totschlagsfredus der Rüstringer 10 Geldmark oder 10 Schillinggewichte Gold oder $2^{2 / 3}$ Mark weißen Silbers betragen, sich also zu dem Normalgelde, das sich auf 40 Geldmark belief, genau wie 1:4 verhalten.

Das "Geld" des Brokmerbriefs betrug 8 höchste Mark oder 96 höchste Schillinge ${ }^{2}$ ), und als Friedensgeld hatte der Totschläger an die Leute 2 höchste Mark zu zahlen. ${ }^{3}$ ) Auch

gestellten Missetaten mit dreifacher Buße belegt hatte, ersieht man aus $\S 220$ des Brokmerbriefs (Rq. 181), womit Fries. Rq. 81, 10 (Rüstr.) und Hettema, Fiv, Ldrgt. S. 102 zu vergleichen sind.

1) Auch die Buße für Lebensgefährdung, die nach altfries. Rechte eine große Hauptlösung betrug, machte nach der 12. Rüstringer Küre (Rq. 116) 10 Geldmark aus! - ${ }^{2}$ ) Aus $\$ 190$ (hond of ... ên half ield) und $\$ 192$ (thi thuma of ... ên fiardendêl ênes ieldes) folgt, daß die Buße für die vier Finger zusammen eine halbe Handbuße oder $1 / 4$ Geld betrug. Es lautet aber $\$ 197$ : Thera fiuwer fingra alvec ên half merc, sa istera litha alvec tuêne skillingar. Die vier Finger waren also zusammen mit 2 Mark oder 24 Schill. zu büßen, d. b. das Geld belief sich auf 8 Mark oder 96 Schillinge. - ${ }^{3}$ ) Dies ergibt sich aus zahlreichen Strafbestimmungen. So sollte z. B. nach $\$ 70$ (Rq. 161) bei skakrâf an die Leute ein Friedensgeld von 2 höchsten Mark fallen. Durch "violenta rapina (nôtnumfti) ${ }^{*}$ wurde aber in Ostfriesland, wie schon aus Lex Fris. VIII und IX $14 \mathrm{ff}$. ersichtlich, dasselbe Friedensgeld wie durch einen unqualifizierten Totschlag verwirkt! Die Richter erhielten im Brokmerlande als brekma vom Totschläger 1/2 höchste Mark (vgl. z. B. $\$ 181$ Rq. 177, 8), so daB der Verbrecher als Gesamtstrafe (frethe + brekma) $2^{1 / 2}$ höchste Mark zu zahlen hatte. In dem Victorburer Verzeichnis der Strafen, welche durch die Fehde der Westre- und Astrefliute verwirkt waren, ist die Totschlagstrafe und ebenso die Notzuchtstrafe auf 5 Mark, also doppelt so hoch wie beim Gemeinfreien, bemessen. Die verantwortlichen Teilnehmer an dieser Fehde müssen also einem über den Gemeinfreien stehenden Stande angebört haben, d. h. Edelinge gewesen sein. 
hier war also das Friedensgeld dem vierten Teile des Geldes gleich.

Nach allem ist es Heck ebensowenig wie His gelungen, gegen mein Ergebnis, daß nach altostfriesischem Rechte der normale Totschlagsfredus dem vierten Teile des Normalgeldes gleichkam, etwas Stichhaltiges vorzubringen. Dieses Ergebnis steht ebenso unerschütterlich fest wie mein anderes Ergebnis, daß nach friesischem Rechte Notzucht dieselben strafrechtlichen Folgen wie der Totschlag hatte. Was aber aus den beiden Ergebnissen für die Stellung des Szêremon innerhalb der friesischen Ständeverfassung folgt, lält sich durch zwei unanfechtbare logische Schlüsse feststellen:

1. Der Totschlagsfredus war genau so hoch wie die Notzuchtstrafe. Diese machte für den Freien ein halbes Szêremonsgeld aus. Folglich betrug der normale Totschlagsfredus ein halbes Szêremonsgeld.

2. Das Normalgeld betrug viermal so viel wie der normale Totschlagsfredus. Dieser belief sich auf ein halbes Szêremonsgeld. Folglich betrug das Normalgeld das Doppelte des Szêremonsgeldes.

Die Betrachtung der Notzuchtstrafe führt also hinsichtlich des Normalgeldes zu genau demselben Resultate wie die oben angestellte Untersuchung der Notzuchtbuße.

Demnach steht es fest, daß es in Friesland noch um das Jahr 1300 unterhalb der Gemeinfreien einen Stand gegeben hat, dessen Glieder ein halb so hohes Wergeld wie die Gemeinfreien hatten.

Heck hat sich nicht getraut, in eine genaue Erörterung der Brokmer Szêremon-Stellen einzutreten und meinen Gründen und Schlüssen eine geschlossene Beweisführung entgegenzustellen. El begnügt sich, seinem Ärger über meine Nachweise, daß noch im späteren Mittelalter in Friesland ein Stand unterhalb der Gemeinfreien existiert hat, durch eine Reihe aphoristischer Bemerkungen (S. 85-87), die von Unrichtigkeiten strotzen, Luft zu machen ${ }^{1}$ ) und schließlich mit

1) Auch in das Gebiet der Philologie hat sich Heck bei dieser Gelegenheit verstiegen (S. 87 Anm. 1), um mich zurechtzuweisen. Er beanstandet, daß ich den Dialekt der Mittelfriesen als „mittelfriesisch“ bezeichne, weil er nicht weiß, daß ich die sonst übliche, aber leicht 
Emphase zu verkünden: „Relevant wäre die Bußerniedrigung des szêremon gewesen. Sie hat sich als Schein erwiesen." Um aber die Verlegenheit, in welche ihn die Brokmer Szêremon-Stellen gebracht haben ${ }^{1}$ ), zu verhüllen und den Schein, $\mathrm{da} B$ er recht habe, zu retten, entschloß er sich zu einer verzweifelten Taktik. Obwohl er nämlich wußte, daß nur die beiden Brokmer Szêremon-Stellen für die ständische Eingliederung des szêremon entscheidende Bedeutung baben, die beiden Hunsegauer dagegen für diese Eingliederung nicht in Betracht kommen, stellt er, um mir den „Fehler der Teilinduktion" vorwerfen zu können, die Sache gerade umgekehrt dar. Nun passierte ihm in der Hast das Unglück, nicht zu erkennen, daß die erste der beiden "signifikanteren" Hunsegauer Stellen lückenhaft ist. Er verstand sie daher gar nicht. Aber was er herauslas, verführte ihn dazu, mir (S. 81) eine Behauptung unterzuschieben, an welche ich noch niemals gedacht habe und von der sich daher in meinen Aufsätzen kein Wort findet. Nach mir hätte nämlich, so redet Heck seinen Lesern vor, "die Frau nur $1 / 2 \times 3 / 2=3 / 4$ Buße des standesgleichen Mannes beanspruchen können". „Jaekel gibt zwar nicht die Ziffer, aber er hat den Mut, diese Ansicht wirklich zu vertreten." Es wäre für mich interessant zu erfahren, wo ich eine solche Ansicht vertreten habe! Gegen diese mir schlankweg untergeschobene Ansicht führt Heck (S. 80-85) ein starkes Aufgebot von Zitaten und eine ihm von Th. Siebs gelieferte Etymologie des Wortes szeremon ins Feld und, nachdem er in diesem Kampfe gegen Windmühlen Sieger geblieben, verkündet er: „Jaekel hat sich geirrt."

mißverständliche Bezeichnung dieses Dialektes als "westfriesisch" grundsätzlich vermeide. Ebenda doziert er: „In den Worten 'respondere pro terra nec pro servis letari nec pro meitele' ist 'letari' der unflektiert übernommene Nominativ Plural., die friesische Bezeichnung der servi." Mit dem friesischen Nominativ Plural. ist er, wie man sieht, noch immer nicht ins reine gekommen.

1) Durch folgerichtiges Schließen hätte er von seiner Annahme aus, daß der Totschlagsfredus ${ }^{3} /{ }_{10}$ Geld betragen habe, zu dem Ergebnis gelangen müssen, daß das Geld des Szeremon $3 / 5$ vou dem des Gemeinfreien betragen habe. Aber der Entdecker der friesischen Standeseinheit hätte sich dam selbst ad absurdum geführt. 
Diesem Manöver gegenüber will ich mich darauf beschränken,

1. bestimmt auszusprechen, was meiner Ansicht nach das altostfriesische Recht über das Wergeld und über die Beweispflicht des freien Weibes bestimmte,

2. die beiden Hunsegauer Szêremon-Stellen zu erläutern,

3. die von Siebs gegebene Etymologie des Wortes szeremon zu beleuchten.

Das Wergeld des Weibes betrug, wie ich behaupte, nach dem ältesten Rechte der Ostfriesen genau die Hälfte von Wergelde des standesgleichen Mannes, das Wergeld des freien Weibes also halb soviel wie das des freien Mannes, d. h. das gemeinfreie Weib hatte das Wergeld des Liten bezw. des Szêremons. Mit den Bußen verhielt es sich genau ebenso. Entsprechend brauchte das gemeinfreie Weib, wenn es auf eine Buße Anspruch machte, keinen stärkeren Beweis zu erbringen als der Szêremon.

Das Weib gehörte zu den "wehrlosen " Leuten. Diese waren gegen Angriffe von seiten Wehrhafter durch einen Sonderfrieden (îfrethe) geschütat ${ }^{1}$ ), und es hatte dieser den "Schwachen" gewährte Sonderfrieden in Ostfriesland allgemein die Verdreifachung der Wergelder, Bußen und Friedensgelder und die Verdreifachung der Zahl der eventuell zu schwörenden Reinigungseide zur Folge. Wurde eine freie Frau von einem Manne angegriffen oder erschlagen, so trat jener Sonderfriede in Kraft, so war, wie es $\$ 34$ der jüngeren Langewolder Küren ausdrückt, die Buße dreifach. ${ }^{2}$ ) Die Frau hatte dann eine dreimal so hohe Bube oder dreimal soviele Reinigungseide als sonst, d. h. solange die Bedingungen für diesen Sonderfrieden nicht gegeben waren, zu beanspruchen. Es mußte ihr in diesem Falle, weil sie in Buße und Wergeld dem Szêremon gleichstand, dreimal soviel Buße und Recht als dem Szêremon oder anderthalbmal soviel Buße und Recht wie dem Manne, d.i. dem freien Manne, zuteil werden. Die zahlreichen ostfriesischen An-

1) Durch "Rechtsfriede" (Heck S. 83) darf man âfrethe natürlich nicht übersetzen. - ${ }^{2}$ ) Fries. Rq. 372: „Waerso men eene vrouwe dulget dat is wondet of van den lyve doet, binnen den huuse of bûten op dat velt, is de boete drêrolt." 
gaben über die Überbuße und das Überrecht des freien Weibes, welche Heck als "Kontrollnachrichten" zusammengetragen und die z. T. auch His angeführt hat ${ }^{1}$ ), stimmen mit meiner Auffassung genau überein. Alle besagen, daß die Frau anderthalbmal soviel Buße und Recht als der mon, keine einzige, daß sie anderthalbmal soviel Buße und Recht als der szêremon erhalten soll.

Die Gewährung der Überbuße und des Überrechts fand ihre folgerichtige Ergänzung darin, daß die Beweispflicht der Frau nicht erhöht wurde. Das Beweisrecht der freien Frau blieb, mochte durch ihre Verletzung ihr Sonderfriede gebrochen sein oder nicht, das des Szêremons. Daß sie prinzipiell in der Beweispflicht dem mon, also dem freien Manne, gleichgestanden habe, wie Heck wähnt, ist nirgends gesagt. Fine solche Gleichstellung fand nur dann statt, wenn die Frau der angreifende Teil gewesen war. Dagegen spricht ihr die einzige Stelle, welche über diese Beweispflicht klare Auskunft gibt, \& 62 der Hunsegauer Bu(Btaxen ${ }^{2}$ ), gleich im ersten Satze ganz allgemein das Beweisrecht des Szêremons zu: Thiu frowa hire lithe mith nôwet ti mâra riuchte ni âch on ti lêdane sa thi stêrmon, d. i.: „Die Frau braucht ihre Gliedmaßen nicht nach höherem Beweisrechte einzuklagen als der Szêremon."

Der Sonderfriede gewährte also eine zweifache Wohltat: er gab einerseits der verletzten Frau Überbuße und Überrecht und gestattete ihr andrerseits, die auf das Dreifache erhöhte Buße mit derselben Zahl von Eiden wie die einfache Buße zu gewinnen. Die freie Frau erhielt daher, wenn ihr afrethe gebrochen war, anderthalbmal soviel Buße und Recht als der freie Mann, während ihre Beweispflicht die des Szêremons blieb.

Beschuldigte man die Frau, daß sie ihren âfrethe durch Kampf verloren habe, so konnte sie ihn mit einem Eide behaupten. Bezichtigten sich der Mann und die Frau gegenseitig des Kampfes, so durfte sie sich zuerst eidlich reinigen und wahrte dadurch ihren $\hat{a}$ frethe. Grab sie aber zu, daB der Mann in der Gegenwehr gehandelt habe ${ }^{3}$ ), so sollte sie in

1) Zeitschrift XXVIII S. 443. - 2) Fries. Rq. 339 - ${ }^{3}$ ) Siebs druckt (bei Heck S. 76) das verderbte handschriftliche ief hiu him ouder dede 
Buße und Recht nichts vor ihm voraushaben (\$62). Wenn dagegen zwei Frauen miteinander kämpften, so konnte keine von ihnen den Sonderfrieden brechen, weder den kleineren noch den größeren ( $\$ 46)$. Dann standen jeder von beiden selbstverständlich Buße und Recht des Szêremons zu.

Im Hinblick auf diese Doppelwirkung des dem Weibe gesetzten Sonderfriedens wird man die Erklärung, welche $\S 45$ der Hunsegauer Bußtaxen von diesem Sonderfrieden gibt, für lückenhaft erklären müssen, denn man vermißt in ihr eine Äußerung über die Nichterhöhung der Beweispflicht des Weibes. Dab diese Erklärung nicht lückenlos überliefert sein kann, geht aber auch daraus hervor, daß sie in der Fassung, in welcher sie uns erhalten ist, einen handgreiflichen Unsinn aussagt. Die Erklärung lautet in den beiden Handschriften:

Thet is to âfrethe, tha ma hire thrimine further bêta skele iefta biriuchta tha êne sxêremonne alsa hit edeth.

Heck und His wollen dies so verstehen: Dies ist der gesetzte Sonderfrieden, daß man ihr anderthalbmal mehr Buße zahlen oder Reinigungseide schwören soll als einem Szeremon, wenn er es getan hat.

Hier tritt also der Szêremon als Täter auf. Eine Frau konnte aber selbstverständlich nur dann Buße oder eine eidliche Reinigung fordern, wenn ihr etwas geschehen, wenn sie also nicht die Täterin war. Wollte man die Stelle mit Siebs, Heck und His so, wie sie überliefert ist, hinnehmen, so würde sie besagen, daß die verletzte Frau anderthalbmal soviel Buße oder Reinigungseide wie der Täter zu beanspruchen habe. Daß damit etwas Widersinniges gesagt wäre, liegt auf der Hand. Dem Täter war weder Buße zu zahlen noch ein Reinigungseid zu leisten, sondern ihm war von der Verletzten der Beweiseid zu schwören, durch welchen diese ihren Bußanspruch begründete. Dabei sollte die verletzte Frau, wie wir aus $\$ 62$ der Hunsegauer Bußtaxen bestimmt

bikent unverändert $a b$ und übersetzt: „Wenn sie aber ihm zugibt, den Streit angefangen zu haben." Diesen Sinn muB die Stelle nach dem gauzen Zusammenhange haben. Für ouder dede ist onder-dede zu lesen. Dieses Wort ist aus *ondwver-dêde entstanden und bedeutet "Gegenwehr-tat". 
wissen, zu keinem höheren Beweise verpflichtet sein als der Szêremon. Nur dies kann es gewesen sein, was jene Erklärung ausdrücken wollte, als sie den szêremon zur Vergleichung heranzog. Die Bestimmung über die Beweispflicht der Frau kann in dem verlorenen Grundtexte nur dahin gelautet haben, daß die Verletzte dem mon, d. h. dem freien Manne, der sie verletzte, keinen höheren Beweiseid zu schwören brauche, als ihn ein Szêremon zu leisten hatte. Es muß also zwischen biriuchta und tha ene sxêremonne eine Zeile verloren gegangen sein. ${ }^{1}$ ) Nach allem kann jene Erklärung im Grundtexte den der Frau unter gewissen Bedingungen zustehenden Sonderfrieden nur dahin näher bestimmt haben, daß man ihr anderthalbmal mehr als einem freien Manne Buße zahlen oder Reinigungseide schwören solle, daß man ihr aber keinen höheren Beweiseid aufzuerlegen habe als einem Szêremon, "wenn ein solcher es tut", nämlich einen Beweiseid leistet.

Die freie Frau sollte also, wenn sie angegriffen wurde, dreimal soviel Buße und Recht, als sie sonst zu beanspruchen hatte, erhalten, dagegen sollte ihre Beweispflicht nicht höher als die des Szêremons sein. Daraus geht klar hervor, $\mathrm{da} B$ sie auf demselben Wergeldniveau wie dieser stand.

Mit unserer Auffassung des der Frau zustehenden Sonderfriedens sind sämtliche Kontrollnachrichten in genauem Einklang. Sie bestätigen lediglich, was sich aus den SzêremonStellen ergibt, nämlich daß das Wergeld der gemeinfreien Ostfriesin von Hause aus dasselbe war wie das des Szêremons oder Liten. Dieses alte Verhältnis erlitt erst gegen Ende des Mittelalters hier und da Modifikationen. So bemerken die Wurster Willküren von 1508 , die das jechtige Geld eines Mannes und ebenso das einer Frau oder Jungfrau

1) Auf diese Lücke wollte ich hinweisen, wenn ich (Zeitschrift XXVII S. 310) den Schluß der Stelle als "lückenhaft" bezeichnete. Den letzten Satz „alsa hit edeth" übersetzt Siebs: „falls er es getan hat". Aber edeth kann nicht "getan hat" bedeuten. Die von andrer Seite vorgeschlagene Übersetzung, wenn er es tut" findet eine Stütze an der Wendung te riuchtane alsat ên mon det in $\$ 9$ der Hunsegauer Bußtaxen (Rq. 332, 25), doch "tut" lautet im Altfries. nicht edeth, sondern deth. Die Form edeth ist aus $\bar{e}$ deth, d. i. ên deth, verlesen. Der Satz hat zu lauten: alsa hit ên deth "wenn es einer tut*. 
auf twintich stige fresche marck festsetzen ${ }^{1}$ ), am Schluß, daß bis dahin das Geld eines Mannes tein stige fresche marck das einer Frau oder Jungfrau dagegen nur soven stige fresche marck betragen habe. ${ }^{2}$ ) Im Brokmerbrief herrscht natürlich noch das altfriesische Verhältnis. Die Frau soll nach $\S 208$ (Rq. 179) für einfache wifstrêvene 1 Schill., für qualifizierte 4 Schill., für das Abschneiden ihrer Haube ên fiardandêl hiris eynis ieldes als Buße erhalten. ${ }^{3}$ ) Die drei Bußen verhielten sich, wie der Eingang der Emsiger Bußtaxen (Rq. 212) lehrt, wie 1:4:12. Das Geld des gemeinfreien Weibes betrug also 48 Schillinge, während sich das des gemeinfreien Mannes auf 96 schillinge belief (oben S. 86)! Die weiteren Belege dafür, daß das Wergeld des Weibes die Hälfte von dem des standesgleichen Mannes ausmachte, werde ich bei andrer Gelegenheit zusammenstellen. ${ }^{4}$ ) Hier haben wir nur noch zu prüfen, ob Heck wohl daran getan hat, die von Siebs gelieferte Etymologie des Wortes szercmon gegen mich ins Feld zu führen.

Siebs nimmt an ${ }^{5}$, daß szeremon einfach "Mann" im Gegensatze zum Weibe bedeute und ein altes Kompositum *kerlmon sei. Es würde dem mnd. kerleman entsprechen. Wenn sich diese Annahme begründen ließe, so wäre allerdings eine Stütze für Hecks Auffassung des Szeremon gewonnen, denn dann wäre es wenigstens möglich, daß die ostfriesischen Rechtsquellen unter szcremon dasselbe wie unter mon verstanden hätten. Aber die für jene Etymologie vorgebrachten Gründe sind nichts weniger als stichhaltig. Für das Altostfriesische den Übergang von *kerlmon oder *kerlemon zu szeremon anzunehmen, ist schlechterdings unmöglich. Was will es den altfriesischen Namensformen Kerl und Kerlemon und der Hunsegauer, Emsiger und mittelfriesischen

1) Pufendorf, Observationes iuris universi, 1756, III. app. p. 114. - 2) A. a. O. p. 118. - 3) Diese Stelle scheint His nicht zu kennen. Nur so kann ich mir einigermaßen erklären, daß er sich (S. 443) zu der Behauptung versteigt: "So sagt z. B. gerade der Brokmerbrief $\$ 181$ (Rq.177): das Wergeld des Weibes ist das anderthalbfache Wergeld des Mannes." Dies steht nicht in diesem noch in sonst einem Paragraphen des Brokmerbriefs. - *) In meiner Geschichte des altfriesischen Geldwesens. - ${ }^{5}$ ) Vgl. Siebs bei Heck S. $79 \mathrm{f}$. 
Bezeichnung des Hausmanns als hûs-kerl gegenüber besagen, daß in ein paar mittelfriesischen Urkunden aus dem Schlusse des 15. Jahrhunderts und im Neuostfriesischen auch assibilierte Formen (tzerl, tzirl, tjerl usw.) auftreten? Daraus folgt doch weiter nichts als daß die Assibilierung bei diesem Worte im Altfriesischen noch nicht eingetreten ist. Daß schon das Altfriesische neben kerl die assibilierte Form gekannt habe, wie man nach Siebs' Worten "Freilich erscheint auch die altfriesische Form -kerl" annehmen muß, ist nicht richtig. Siebs möchte die Form kerl auf die $a$-Form (nord. karl) zurückführen oder noch lieber als niederdeutsche bezw. niederländische Entlehnung betrachten. Es sei begreiflich, daß bei einem so geläufigen Worte das plattdeutsche Kerl verdrängenden Einfluß üben konnte. So begreiflich scheint mir diese Entlehnung bei einem so geläufigen Worte nun gerade nicht zu sein. Wann soll es denn zu dieser Entlehnung gekommen sein?

Ganz unerklärlich wäre bei der Annahme, daß szeremon aus *kerlmon oder *kerlemon entstanden sei, das Schwinden des $l$, zumal es doch in dem Eigennamen Kerlemon erhalten blieb. Soll man sich wirklich vorstellen, daß der ostfriesisehe Eigenname Kerlemon und das altostfriesische Appellativum szeremon ein und dasselbe Wort seien? Siebs meint, das Schwinden des $l$ (oder Reduktion des $l$ zu $e$ ) sei in solchem Falle im Altfriesischen gar nicht außerordentlich, und verweist auf skemma (sonst skel ma "soll man"). Aber daß in einer nicht hochtonigen Silbe ein $l$ einem folgenden $m$ assimiliert oder $l$ zwischen $r$ und $m$ zu $e$ reduziert worden sei, dafür fehlt es im Altfriesischen an Belegen.

Nach allem ist mir Siebs' Behauptung, daß bei seiner Etymologie "keine lautlichen Schwierigkeiten bestehen", nicht verständlich. Die ostfriesische Namensform Kerlemon ist für sich allein Beweis genug, daß das altostfriesische Wort szerenon nicht aus *kerlemon entstanden sein kann. Wahrscheinlich haben Hecks sachliche Ausführungen Siebs so stark eingeleuchtet, daß er die lautlichen Schwierigkeiten, die sich bei seinem Erklärungsversuche ergaben, übersah. ${ }^{1}$ )

1) Die Versuche, das Wort szeremon zu deuten, werden hinfort von der Tatsache auszugehen haben, daß der Szeremon unter dem 
Etymologische Gründe lassen sich demnach für Hecks Gleichsetzung des szeremon mit dem mon nicht geltend machen. Der Versuch, meine Auffassung des friesischen szeremon als irrig zu erweisen, ist somit nach jeder Richtung gescheitert.

\section{§ 5. Das spätere friesische Normalwergeld und die Wergeldbeträge der Lex Frisionum.}

Nach der ,siegreichen“ Niederkämpfung meiner auf exakte Beweise gestützten Ansicht, daß sich die alte ständische Tripartitio der Friesen das Mittelalter hindurch erhalten habe, kehrt Heck seine Waffen gegen meine Behauptung, daß die Normalkomposition der späteren friesischen Rechtsquellen nicht mit der Edelings-, sondern mit der Freienkomposition der Lex Frisionum zusammenfalle, und betritt damit das Gebiet der friesischen Numismatik, auf dem er sich von jeher heimisch fühlte.

In einer langen Einleitung, die Richtiges und Falsches durcheinander mischt (S. 103-110), weist er auf die enormen Schwierigkeiten und Gefahren seines Vorhabens hin, um dieses dann endlich auf 68 Seiten (S. 110-177) zur Ausführung zu bringen. In Wirklichkeit ist die Feststellung, ob sich das spätere friesische Normalgeld mit dem Freienoder mit dem Edelingsgelde der Lex Frisionum deckt, für einen in den friesischen Rechtsquellen bewanderten Münzmetrologen eine nicht allzu schwere Aufgabe, die sich bequem

Gemeinfreien stand, ein halb so hohes Wergeld wie dieser hatte. Sollte es sich dabei als möglich herausstellen, das Wort als rein deutsch zu erweisen, so werde ich nichts einwenden, wenn man die von mir gegebene Etymologie als "Ungeheuerlichkeit" bezeichnet. Bis dahin aber muß ich daran festhalten, daß szeremon = szêre-mon und dieses aus *cere-mon, einer hybriden Bildung aus lat. cera "Wachs" und fries. mon "Mann", entstanden ist, also den Wachszinsigen (cerarius, cerearius) bezeichnet. Natürlich könnte man das anlautende $s x$, $s z$ auch auf $s k$ (statt auf $k$ ) und so szeremon auf *scarimon zurückfübren, den Szeremon also für einen "Dienstmann" erklären. Aber auch bei dieser Erklärung käme der Szeremon auf das Litenniveau zu stehen. Die Identifizierung des szeremon mit dem keremon des Brokmerbriefs § 212 (His in Zeitschr. XXVIII S. 443) ist aus sprachlichen und sachlichen Gründen abzuweisen. 
auf zwei Seiten lösen läßt. Soll z. B. das ostfriesische Normalgeld zu 40 Geldmark mit den ostfriesischen Kompositionen der Lex verglichen werden, so ist nichts weiter nötig, als das Silberquantum, welches 40 Geldmarken enthielten, zu bestimmen und in die Münznominale der Lex umzurechnen. Scheut sich Heck, an die Lösung dieser einfachen Aufgabe zu gehen, um nicht mit eigener Hand seine Ständetheorie zu untergraben? Oder ist ihm wirklich die Lösung dieser Aufgabe zu schwer? Das letztere ist in der Tat der Fall. Heck vermag trotz seiner "Kenntnis" der friesischen Rechtsquellen und des friesischen Münzwesens nicht einmal den Silberwert der Geldmark festzustellen, und nur, um dies zu verbergen, erregt er den Schein, als ob es sich um eine furchtbar schwierige Aufgabe handele, der sich nur durch eine raffinierte Methode beikommen lasse, die erst von ihm ausgebildet und mit Erfolg angewandt worden sei. Daher unterläßt er es auch, mit klaren Worten die Frage nach dem Silberwert der Geldmark zu stellen und zu prüfen, ob die friesischen Rechtsquellen eine exakte Beantwortung dieser Frage gestatten oder nicht. Er sucht vielmehr durch Exkurse ${ }^{1}$ ) und Hypothesenbauten die Aufmerksamkeit des Lesers von jener Kernfrage der ganzen altfriesischen Münzmetrologie und der ganzen Kontroverse über Fortdauer oder Verschwinden der alten ständischen Tripartitio abzulenken. Die Frage gar nicht zu berühren war natürlich auch für ihn unmöglich. So liest man denn endlich über seinem $§ 11$ die

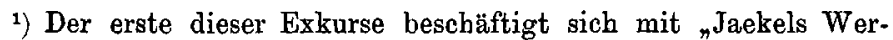
geldtheorie" ( $\$ 8 \mathrm{~S}$. $112 \mathrm{ff}$.) und sucht zu widerlegen, was ich in meinem Aufsatze "Êtbeling, Frîmon, Frîling und Szêremon" über den Sinn, in welchem die ältesten friesischen Rechtsquellen das Wort "Wergeld" gebrauchen, ausgeführt habe. Meine Abhandlung "Weregildus und simpla compositio" hat Heck beim Druck dieses Exkurses zwar schon gekannt, aber - wohl aus taktischen Gründen - beiseite geschoben. Soweit sein Exkurs durch diese Abhandlung nicht schon erledigt ist, wird ex demnächst an einer anderen Stelle gewürdigt und dabei die Beweisführung Hecks näher geprüft werden. - Der zweite Exkurs (\$9 S.126ff.) bespricht "die Umrechnung der Rednathespfennige". Die zahlreichen Irrtümer, welche hier hinsichtlich der friesischen Münzen des 11. Jahrhunderts vorgetragen werden, sollen in meiner Geschichte des friesischen Geldwesens eingehend besprochen werden. Dabei wird auch die Hecksche Beweisführung zu beleuchten sein. 
Überschrift: „Der Silberwert der Geldmark.“ Aber unmittelbar vorher macht Heck die charakteristische Bemerkung: „Der Streit über den Silberwert der Geldmark hat ... für die Endfrage (hierunter versteht er die Frage, ob das spätere friesische Normalgeld dem Edelings- oder dem Freiengelde der Lex entspreche,) nur geringe Bedeutung. Wer sich nur für die Endfrage interessiert, kann den $\S 11$ völlig überschlagen. Ich füge ihn der Vollständigkeit halber und deshalb ein, weil Jaekel auch bei dieser Frage grundlose Angriffe gegen meine Arbeitsmethode erhoben hat." Nach Heck schadet es also nichts, wenn man seinen $\$ 11$ einfach überschlägt. Er hat auch hierin vollkommen recht, denn er hat in dem ganzen $\& 11$ auch nicht ein Wort über den Silberwert der Geldmark verloren, sondern lediglich einen - total mißglückten - Versuch gemacht, seine Berechnung der Geldmark zu 120 statt zu 144 Rednathpfennigen als richtig zu erweisen. ${ }^{1}$ ) Statt den Silberwert der Geldmark aus positiven Quellenangaben zu ermitteln und von diesem festen Grunde aus die Wergeldvergleichung vorzunehmen, wagt er, um seine Ständetheorie zu retten, die verzweifelte Behauptung, daß die Geldmark 8 fränkische Goldtriente wert gewesen sei; und da sich in den friesischen Rechtsquellen auch nicht der Schatten eines Beweises für diese Behauptung entdecken läßt, spiegelt er dem Leser Beweise vor, indem er aus teils unrichtigen, teils schief gefaßten Sätzen über einigen rein

1) Hecks Deduktion leidet an einem grundstürzenden Fehler. $\mathrm{Er}$ geht nämlich von der irrigen Annahme aus, daß in den Allgemeinen Bußtaxen mit der Geldmark derselbe Wert gemeint sei wie mit der "Mark" schlechthin. Diese "Mark", die einen ganz anderen Wert als die Geldmark hatte, zerfiel in 120 Weißpfennige. Auf sie, nicht aber auf die Geldmark, finden Hecks Ausführungen in seinem $\$ 11$ und die Bemerkungen ron His (Zeitschr. XXVIII S.439f.) Anwendung. Die beiden Forscher haben von der Entstehung der Allgemeinen Bußtaxen keine Vorstellung und haben sich von der verfehlten Annahme, die ich hinsichtlich der Mark und der Geldmark in meinem vor einem Vierteljahrhundert geschriebenen und von Heck verurteilten Aufsatze über das friesische Pfund und die friesische Mark aufgestellt habe, noch immer nicht losgemacht. Daß die Geldmark jemals auf 40 Schillinge cona veranschlagt und zu 10 Schillingen gerechnet worden sei, ist nieht „unbestritten und völlig sicher", sondern bestimmt falsch.

Zeitschrift für Rechtsgeschichte. XXX. Germ. Abt. 
willkürlichen Annahmen einen gewaltigen Hypothesenbau aufführt und dann kühn behauptet, „den abschließenden Beweis für die frühere Rechnung der Geldmark zu 8 Trienten und für die neue Rechnung der Geldmark zu 10 Schillingen" erbracht zu haben. Eine nähere Besichtigung dieses luftigen Hypothesenbaus wäre Zeitverschwendung. Wir beschränken uns darauf, der Heckschen Behauptung, daß die Geldmark 8 fränkische Goldtriente gegolten habe, dadurch den Boden zu entziehen, daß wir den Silberwert der Geldmark quellengemäß feststellen.

Um den Silberwert der Geldmark zu bestimmen, braucht man weder von ihrer Einteilung noch von dem ihr zugrunde liegenden Rednath- oder Kawingpfennige etwas zu wissen, sondern nur die Angaben zu beachten, welche das im 14. Jahrhundert abgefaßte Fivelgauer Stück ,fon ielde" über die sukzessive Erhöhung der Normalkomposition macht. ${ }^{1}$ ) Im Fivelgau und im Hunsegau wurde im 13. Jahrhundert das Normalgeld, das bis dahin 40 Geldmark oder 480 Reilmark ausgemacht hatte, bedentend erhöht. Der Umschwung, der um dieselbe Zeit in der Geldrechnung eintrat, führte dann dahin, daß man die in den kleinen einheimischen Marken ausgedrückte Komposition in schwerere Marksorten umrechnete. Infolge dieser Umrechnung wurde das Normalgeld der Hunsegauer und Fivelgauer, wie die Hunsegauer Küren von 1252 (Fries. Rq. 328), die Küren von Fivelgau und Hunsegau (Rq.,301) und die Fivelgau-Oldamter Küren (Rq. 298) unmittelbar ergeben, schließlich zu 16 Mark weißen Silbers oder 16 Mark Englisch angesetzt. Aus jener Fivelgauer Aufzeichnung "fon ielde", deren Verfasser nach der Groninger Mark und dem Groninger Pfunde rechnet, erfahren wir nun, wieviel die Wergelderhöhung des 13. Jahrhunderts betrug. Sie berichtet nämlich, daB durch sie das forme ield von 24 auf 36 Mark und entsprechend das ield von 240 auf 360 Mark stieg. Das "Greld" sei dann zu 20 Höchsten Mark, die Mark zu 12 Pfunden Groninger Gepräge gerechnet, angesetzt worden, und diese 20 Mark seien gleich 16 Mark Englisch. Man hatte also die 360 Mark, auf welche das Geld erhöht worden war, in

1) Hettema, Fivelg. Ldrgt. S. 130. 132. 
20 Höchste Mark oder 16 Mark Englisch umgerechnet, denn 20 Höchste Mark zu je 12 Pfund Grenengslachta machten, da die Groninger Mark 160 Pfennige umfaßte, $\left(20 \times 12 \times{ }^{3}{ }^{2}=\right)$ 360 Mark Grenengslachta aus. Demnach betrug das neue "Geld" (360 Mark Grenengslachta $=20$ Höchste Mark $=$ 16 Mark Englisch) das Anderthalbfache des älteren „Geldes" (240 Mark Grenengslachta). Die 40 Geldmark oder 480 Reilmark, auf welche sich dieses ältere "Geld" in friesischer Münze belief, müssen also mit $\left(16 \times{ }^{2 / 3}=\right) 10^{2 / 3}$ Mark Englisch oder $10^{2} / 3$ Mark weißen Silbers gleichwertig gewesen sein, so daß 1 Geldmark $={ }^{4 / 15}$ Mark Englisch oder ${ }^{4} / 15$ Mark Silber.

Bestätigt wird dieses Ergebnis durch die 13. Rüstringer Küre, die die Brüche für den pflichtvergessenen Volksbeamten das eine Mal auf 8 Mark Silber, das andere Mal auf 30 Mark, d. i. 30 Geldmark, festsetzt. ${ }^{1}$ ) Auch hieraus folgt: 1 Geldmark $={ }^{4} / 15$ Mark Silber.

Der Silberwert, welcher der Mark Englisch (144 Sterl.) des 13. Jahrhunderts zukam, ist von uns bereits oben (S. $60 \mathrm{f}$.) festgestellt worden. Es ergab sich dort, daß 16 Mark Englisch genau 10 röm. Pfund Silber enthielten. Auf 1 Mark Englisch gingen also $5 / 8$ röm. Pfund Silber oder, da das altfriesische Denargewicht ${ }^{1 / 288}$ röm. Pfund betrug ${ }^{2}$ ), 180 altfriesische Silberpfennige.

Nach allem hatte die Geldmark, die $4 / 15$ Mark Englisch betrug, einen Wert von 48 altfriesischen Silberpfennigen, und der Rednathpfennig (=1/144 Geldmark) machte genau 1/3 vom altfriesischen Silberpfennige aus. Die 40 Geldmark aber, auf welche sich das ostfriesische Normalgeld vor den Wergelderhöhungen des 13. Jahrhunderts belief, waren $48 \times 40$ altfriesischen Silberpfennigen gleich. Rechnet man diesen Betrag nach dem karolingischen Wertverhältnisse der beiden Edelmetalle (12:1) in Gold um, so erhält man $(48 \times 40: 12=) 160$ altfriesische Goldpfennige oder $53^{1 / 3}$ altfriesische Goldschillinge, d. h. die ostfriesische Freiencompositio der Lex Frisionum.

1) Fries. Rq. 116. Vgl. oben S. 86. - ${ }^{2}$ ) Vom altfriesischen Pfenniggewichte (1/2ss röm. Pfund) habe ich bereits Zeitschrift XXVII S. 116f., 283, 287, 303, XXVIII S. 107 gesprochen. 
An diesem Ergebnisse läßt sich nicht rütteln.

Auf Hecks Auslassungen über das mittelfriesische Normalgeld (S. 169ff.) und über die Bedeutung der FrîlingsStellen (S. 177ff.) einzugehen erübrigt sich. Daß sie mit Irrtümern behaftet und ihre Ergebnisse falsch sein müssen, bedarf keines besonderen Beweises mehr, denn diese Auslassungen fußen in letzter Linie auf Hecks unrichtiger Bestimmung der Geldmark.

Wie die vorstehende Untersuchung gezeigt hat, ist Hecks Versuch, seine beiden Behauptungen, daß bei den Friesen im Mittelalter Standeseinheit geherrscht habe und daß nicht die Freien-, sondern die Edelingskomposition der Lex Frisionum in dem Normalgelde der späteren friesischen Rechtsquellen fortlebe, mit den positiven Quellenzeugnisson in Einklang zu bringen, völlig gescheitert. Denselben Mißerfolg würde er $\mathrm{zu}$ verzeichnen haben, wenn er seine alte Behauptung, daß man nicht in dem homo liber, sondern in dem homo nobilis der Lex Frisionum den Gemeinfreien zu sehen habe, ernstlich verteidigen, d. h. beweisen wollte, daß die Compositio, welche der gemeinfreie Friese nach Lex Rib. 36 hatte, mit der Edelingskomposition der Lex Frisionum übereinstimme.

Die Ständetheorie Hecks ist mit den authentischen Nachrichten über die Standesverhältnisse der Friesen absolut unvereinbar.

In der Einleitung zu seiner Abhandlung erzählt uns Heck (S. 52f.), daß er durch sein Studium der friesischen Standesverhältnisse zu seiner Ständetheorie gelangt sei. Bis jetzt hat er aber für seine Auffassung dieser Standesverhältnisse noch nicht einen durchschlagenden Beweis, sondern lediglich mehr oder weniger willkürliche Annahmen geltend zu machen gewußt. $Z$ war behauptet er immer und immer wieder, für seine Auffassung positive Anhaltspunkte gewonnen und von einander unabhängige Gründe von unmittelbarer Beweiskraft beigebracht zu haben, aber bei näherem Zusehen entdeckt man bald, daß seine Gründe in letzter Linie willkürliche Hypothesen sind und seine Argumentationen der zwingenden Kraft entbehren. Er meint, daß kein Forscher auf diesen schwierigen Gebieten ohne Wahrscheinlichkeits- 
annahmen auskommen könne (S. 57). Gewiß muß der Forscher hier und da, wenn positive Quellenangaben fehlen, die Lücke der Überlieferung durch eine Vermutung zu überbrücken suchen, aber er darf nicht eine rein willkürliche Annahme zum Ausgangspunkte seiner Untersuchung machen. Jede historische Forschung hat von positiven Quellenzeugnissen auszugehen und im Verlaufe der Untersuchung nur dort, wo ausdrückliche Angaben nicht zu Gebote stehen, mit Wahrscheinlichkeitsannahmen zu operieren.

Ausgangs- und Mittelpunkt jeder Untersuchung der friesischen Standesverhältnisse muß die Vergleichung der überlieferten Wergeld - und Bußbeträge bilden, und diese Vergleichung darf nicht auf Hypothesen, sondern nur auf bestimmte Zeugnisse und feststehende münzmetrologische Tatsachen gestützt werden. Sie gibt, exakt durchgeführt, sicheren Aufschluß über die vorfränkischen Wergeld- und Standesverhältnisse, über die große Wergeldreduktion des ausgehenden 8. Jahrhunderts, über die kleinen Wergeldänderungen der fränkischen Zeit und über die nachfränkische Wergeld- und Ständeverfassung. Im großen und ganzen stimmen die Ergebnisse dieser Wergeldvergleichung zu der herrschenden Auffassung der friesischen Ständeverhältnisse, wie sie von unseren rechtsgeschichtlichen Autoritäten vertreten wird. Für die genauere Charakteristik des friesischen Ständewesens wird die eine und andere Einzelbeobachtung Hecks verwandt werden können, aber seine Gesamtauffassung dieses Ständewesens ist abzulehnen, weil sie sich mit allen Zeugnissen über die Wergelder des friesischen Stammes in Widerspruch setzt.

Heck hat gegen mich den "Vorwurf der mangelnden Methode" erhoben und in dem Aufbau meiner Schlußfolgerungen "einen erstaunlichen Mangel an Erkenntniskritik" entdeckt. „Jaekel unterschätzt die Irrtumsmöglichkeit. Er sieht nicht genug Möglichkeiten und hält deshalb die erschauten zu Unrecht für allein zulässig. "1) Heck übersieht hier, daß es sich nur um einen Gegensatz zwischen seiner und meiner Darstellungsart handelt. Ich glaube im Verlauf meiner münzmetrologischen Studien noch ein paar Möglichkeiten mehr als er "erschaut“ zu haben, aber während er sämtliche

1) Heck a. a. O. S. 58. 
102 Hugo Jaekel, Die münzmetrologischen Anbaltspunkte etc.

erschauten Möglichkeiten vor dem Leser ausbreitet, pflege ich über die Möglichkeiten, welche ich nach reiflicher Prüfung ausschließen zu müssen glaube, kein Wort zu verlieren, da es mir nicht um den Ruf der Gelehrsamkeit, sondern um rasche Förderung der Erkenntnis des friesischen Altertums zu tun ist. Heck macht mir ferner den Vorwurf der Teilinduktion, der unvollständigen Quellenbeobachtung und holt mehrfach mit lobenswertem Fleiß meine Unterlassung nach. Schade, daß ich für das Beobachtungsmaterial, welches er bei solchen Gelegenheiten zusammengebracht hat, keine Verwendung habe. In meinen Kollektaneen ist es bereits vermerkt. Kollektaneenhefte aber abzudrucken, wie es seit einiger Zeit auf dem Gebiete der friesischen Altertumsforschung Mode geworden ist, läuft meinen Neigungen zuwider. Ich werde nach wie vor auch in der Ständekontroverse von meinen Belegen nur so viele mitteilen, als gerade nötig sind, um meiner Ansicht in jedem wissenschaftlichen Streite, welcher mit ehrlichen Waffen ausgefochten wird, den Sieg zu verbürgen.

Was Heck sonst an Vorwürfen, Unterschiebungen, Entstellungen usw. in seiner Abhandlung bringt, will ich gern seiner Entrüstung zugute halten. Er wird ja selbst, wenn die Beruhigung eingetreten sein wird, manches in anderem Lichte sehen. Überdies bin ich gern bereit, alle Ausstellungen, welche er an meiner Methode gemacht hat, unbesehen als berechtigt anzuerkennen, wenn er den exakten Nachweis führt, daß meine münzmetrologischen Ergebnisse falsch und die seinigen richtig sind. 